\title{
ON THE VARIETY OF PLANE CURVES OF DEGREE $d$ WITH $\delta$ NODES AND $\kappa$ CUSPS
}

\author{
PYUNG-LYUN KANG
}

\begin{abstract}
Let $\mathbf{P}^{N}$ be the projective space which parametrizes all plane curves of degree $d$ and $V(d, \delta, \kappa)$ the subvariety of $\mathbf{P}^{N}$ consisting of all reduced and irreducible plane curves of degree $d$ with $\delta$ nodes and $\kappa$ cusps as their only singularities. In this paper we prove that $V(d, \delta, \kappa)$ is irreducible if $\kappa \leq 3$, except possibly when $\kappa=3$ and $d=5$ or 6 .
\end{abstract}

\section{INTRODUCTION}

In this paper we study the locus $V(d, \delta, \kappa)$ of reduced and irreducible plane curves of degree $d$ with $\delta$ nodes and $\kappa$ cusps as their only singularities.

Let $\mathbf{P}^{N}$ be the projective space parametrizing plane curves of degree $d$, and $V_{d, g}$ the locally closed subset of $\mathbf{P}^{N}$ of reduced and irreducible plane curves of degree $d$ and geometric genus $g$. Harris [H1] recently proved that the variety $V_{d, g}$ is irreducible. Knowing this fact, Diaz and Harris [DH1] showed that

(1.1) If $W \subset V_{d, g}$ is any subvariety of codimension 1 , and $D \in W$ a general point, then the singularities of $D$ are either (1) $m$ nodes, (2) $m-1$ nodes and one cusp (CU), (3) $m-2$ nodes and one tacnode (TN), or (4) $m-3$ nodes and one ordinary triple point (TR), where $m=\frac{1}{2}(d-1)(d-2)-g$.

They asked in [DH2] whether those loci are irreducible. In this paper we prove that the variety $V(d, \delta, 1), \mathrm{CU}$ in (1.1), is irreducible. We essentially follow the same idea as that in [H1]; first we show $V\left(d, \frac{1}{2}(d-1)(d-2)-1,1\right)$, the locus of reduced and irreducible rational plane curves of degree $d$ with $\frac{1}{2}(d-1)(d-2)-1$ nodes and one cusp, is irreducible; second we show that there exists only one component of $V(d, \delta, 1)$ which contains

$$
V\left(d, \frac{1}{2}(d-1)(d-2)-1,1\right)
$$

in its closure; finally we prove that every component of $V(d, \delta, 1)$ admits a degeneration to $V\left(d, \frac{1}{2}(d-1)(d-2)-1,1\right)$.

Using the same argument as above we prove the more general theorem.

Received by the editors April 4, 1988.

1980 Mathematics Subject Classification (1985 Revision). Primary 14H10.

Key words and phrases. Plane curves, cusps, families of plane curves with nodes and cusps. 
(1.2) Theorem. The variety $V(d, \delta, \kappa)$ is irreducible if $\kappa \leq 2$. If $\kappa=3$, then, except possibly when $d=5$ or $6, V(d, \delta, \kappa)$ is irreducible.

Remark. Ziv Ran has recently and independently shown that $V(d, \delta, 1)$ is irreducible. He has also shown that the two other divisors TN and TR of $V_{d, g}$ in (1.1) are irreducible.

(1.3) Definitions and notations. We mean by the (geometric) genus $g(C)$ of a reduced curve $C$ the genus of its normalization; in particular, if $C$ has irreducible components $C_{1}, C_{2}, \ldots, C_{k}$, then

$$
g(C)=\sum_{i} g\left(C_{i}\right)-k+1
$$

If $C$ is a reduced plane curve, then the class $c(C)$ of $C$ is the degree of the dual curve $C^{*}$ in $\left(\mathbf{P}^{2}\right)^{*}$, the projective plane of lines of $\mathbf{P}^{2}$. If a reduced plane curve $C$ has $\delta$ nodes and $\kappa$ cusps as its only singularities, then $g(C)=$ $\frac{1}{2}(d-1)(d-2)-\delta-\kappa$ and $c(C)=d(d-1)-2 \delta-3 \kappa$.

By a nice curve with given singular points we mean a curve all other singularities of which besides given ones are nodes.

Acknowledgment. I would like to thank my thesis advisor Dr. Steven Diaz for his helpful advice and careful proofreading throughout the preparation of this paper.

In this section we prove the first two claims before (1.2) in the Introduction. (2.1) Theorem. Assume $d \geq 3$. Then $V\left(d, \frac{1}{2}(d-1)(d-2)-\kappa, \kappa\right) \subset V_{d, 0}$ is irreducible if $\kappa \leq \min \left(d-2, \frac{1}{2}(d+1)\right)$.

Proof. Note that any rational plane curve of degree $d$ can be realized as a projection $\pi_{\Lambda}$ of a rational normal curve $C$ in the projective space $\mathbf{P}^{d}$ from a $(d-3)$-plane $\Lambda$.

Fix a rational normal curve $C$ and a projective plane $\mathbf{P}^{2}$ to project onto once and for all. Let $\operatorname{Gr}\left(d-3, \mathbf{P}^{d}\right)$ be the Grassmannian of $(d-3)$-planes of $\mathbf{P}^{d}$ and $\mathscr{V}$ the dense subset in $\operatorname{Gr}\left(d-3, \mathbf{P}^{d}\right)$ of $(d-3)$-planes which occur in the above fact and avoid the fixed projective plane. Then we have a map $\pi$ from $\mathscr{V}$ to $V_{d, 0}$ modulo $\operatorname{Aut}\left(\mathbf{P}^{2}\right)$ defined by $\pi(\Lambda)=\pi_{\Lambda}(C)$ for $\Lambda \in \mathscr{V}$. It is therefore enough to show that $\pi^{-1}\left(V\left(d, \frac{1}{2}(d-1)(d-2)-\kappa, \kappa\right)\right)$ is irreducible in $\operatorname{Gr}\left(d-3, \mathbf{P}^{d}\right)$. In fact, since any automorphism of $C$ is given by an automorphism of $\mathbf{P}^{d}$, we have $\pi_{\Lambda}(C)=\pi_{\Lambda^{\prime}}(C)$ if and only if $\Lambda=A\left(\Lambda^{\prime}\right)$ where $A$ lies in $\operatorname{Aut}(C) \subset \operatorname{Aut}\left(\mathbf{P}^{d}\right)$.

Let $\Lambda \in \pi^{-1}\left(V\left(d, \frac{1}{2}(d-1)(d-2)-\kappa, \kappa\right)\right)$. So $\Lambda$ meets $\kappa$ distinct tangent lines, say, $t_{i}$ of $C$ away from $C$, where $t_{i}$ are tangent lines of $C$ at $p_{i} \in$ $C$, for $i=1, \ldots, \kappa$. Let $\sigma_{2}\left(t_{i}\right)=\left\{(d-3)\right.$-planes meeting $\left.t_{i}\right\}$ which is a codimension two Schubert variety of $\operatorname{Gr}\left(d-3, \mathbf{P}^{d}\right)$ (see $[\mathrm{GH}]$ for notations). 
Then $\Lambda \in \bigcap_{i=1}^{\kappa} \sigma_{2}\left(t_{i}\right)$ and a general $(d-3)$-plane in $\bigcap_{i=1}^{\kappa} \sigma_{2}\left(t_{i}\right)$ meets only $\kappa$ distinct tangent lines, otherwise (i.e. if it meets more than $\kappa$ distinct tangent lines of $C$ ) it will be a general member of a subvariety with higher codimension (cf. [EH, Theorem (2.3)]).

Let $C_{\kappa}$ be the $\kappa$ th symmetric product of $C$ and $\Delta$ be the diagonal of $C_{\kappa}$, i.e., $\Delta=\left\{\left(p_{1}, \ldots, p_{\kappa}\right) \in C_{\kappa} \mid p_{i}=p_{i}\right.$ for some distinct $i$ and $\left.j\right\}$. Note that $C_{\kappa} \backslash \Delta$ is irreducible. Since, from what we observed before,

$$
\pi^{-1}\left(V\left(d, \frac{1}{2}(d-1)(d-2)-\kappa, \kappa\right)\right)
$$

can be considered as a fiber space over $C_{\kappa} \backslash \Delta$ with a fiber over a point $\left(p_{1}, \ldots\right.$, $\left.p_{\kappa}\right)$ in $C_{\kappa} \backslash \Delta$ an open and dense set of $\bigcap_{i=1}^{\kappa} \sigma_{2}\left(t_{i}\right)$ consisting of $(d-3)$-planes which meet $t_{i}$ transversely away from $C$, it is enough to show that $\bigcap_{i=1}^{\kappa} \sigma_{2}\left(t_{i}\right)$ is irreducible and of constant dimension. For this purpose we now fix for a while $\kappa$ distinct points $p_{1}, \ldots, p_{\kappa}$ on $C$ and the tangent lines $t_{i}$ of $C$ at $p_{i}$. Let $x_{i} \in t_{i}, i=1, \ldots, \kappa$, and $\sigma_{3}\left(x_{i}\right)$ be the Schubert variety of $(d-3)$-planes containing $x_{i}$ which is codimension three in $\operatorname{Gr}\left(d-3, \mathbf{P}^{d}\right)$. Consider $\bigcap_{i=1}^{\kappa} \sigma_{3}\left(x_{i}\right)$ consisting of $(d-3)$-planes which contain the plane $S_{\kappa}$ spanned by $x_{1}, \ldots, x_{\kappa}$, then $\bigcap_{i=1}^{\kappa} \sigma_{3}\left(x_{i}\right) \subset \bigcap_{i=1}^{\kappa} \sigma_{2}\left(t_{i}\right)$. On the other hand, any $\Lambda \in \bigcap_{i=1}^{\kappa} \sigma_{2}\left(t_{i}\right)$ will lie in $\bigcap_{i=1}^{\kappa} \sigma_{3}\left(x_{i}\right)$ where $x_{i}=\Lambda \cap t_{i}$. We therefore have

$$
\bigcup_{\left(x_{1}, \ldots, x_{\kappa}\right) \in t_{1} \times \cdots \times t_{\kappa}} \bigcap_{i=1}^{\kappa} \sigma_{3}\left(x_{i}\right)=\bigcap_{i=1}^{\kappa} \sigma_{2}\left(t_{i}\right) .
$$

We can now consider in $\mathscr{V} \bigcap_{i=1}^{\kappa} \sigma_{2}\left(t_{i}\right)$ as the fiber space over $t_{1} \times \cdots \times t_{\kappa}$ the product of $\kappa$ lines with a fiber $\bigcap_{i=1}^{\kappa} \sigma_{3}\left(x_{i}\right)$ over a point $\left(x_{1}, \ldots, x_{\kappa}\right) \in$ $t_{1} \times t_{2} \times \cdots \times t_{\kappa}$, i.e., each fiber consisting of general member of $\bigcap_{i=1}^{\kappa} \sigma_{3}\left(x_{i}\right)$. We claim that $\bigcap_{i=1}^{\kappa} \sigma_{3}\left(x_{i}\right)$ is a nonempty irreducible subvariety of $\operatorname{Gr}\left(d-3, \mathbf{P}^{d}\right)$ of dimension $3(d-\kappa-2)$ whenever $d-\kappa-2 \geq 0$ and $2 \kappa-2 \leq d-1$. To see this, choose $\left(x_{1}, \ldots, x_{\kappa}\right) \in t_{1} \times \cdots \times t_{\kappa}$. Then we claim that $\left\{x_{1}, \ldots, x_{\kappa}\right\}$ spans a $(\kappa-1)$-plane $S_{\kappa}$. Suppose not, then $\left\{x_{1}, \ldots, x_{\kappa}\right\}$ would span at most a $(\kappa-2)$-plane and together with $p_{1}, \ldots, p_{\kappa}$ at most a $(2 \kappa-2)$-plane since we may assume that no $x_{i}$ is on $C$, which meets $C$ at $2 \kappa$ points counting multiplicities. But any $n$-plane, $n \leq d-1$, meets a rational normal curve of degree $d$ in at most $n+1$ points. This gives a contradiction once we have $2 \kappa-2 \leq d-1$. Call $S_{\kappa}$ the $(\kappa-1)$-plane spanned by $x_{1}, \ldots, x_{\kappa}$. Considering the projection $\pi_{S_{\kappa}}: \mathbf{P}^{d} \rightarrow \mathbf{P}^{d-\kappa}$ from $S_{\kappa}$ we can easily check that $\bigcap_{i=1}^{\kappa} \sigma_{3}\left(x_{i}\right) \simeq \operatorname{Gr}\left(d-\kappa-3, \mathbf{P}^{d-\kappa}\right)$, which confirms that $\bigcap_{i=1}^{\kappa} \sigma_{3}\left(x_{i}\right)$ is irreducible of dimension $3(d-\kappa-2)$. (Note that $\bigcap_{i=1}^{\kappa} \sigma_{3}\left(x_{i}\right)=\left\{S_{\kappa}\right\}$ when $d-\kappa-3=-1$.) Since the base space $t_{1} \times \cdots \times t_{\kappa}$ and the fibers are irreducible, so is the total space $\bigcap_{i=1}^{\kappa} \sigma_{2}\left(t_{i}\right)$. Q.E.D.

(2.2) Theorem. Assume that $\kappa \leq \min \left(d-4, \frac{1}{2}(d-1)\right)$. Then there exists only one component of $V(d, \delta, \kappa)$ which contains $V\left(d, \frac{1}{2}(d-1)(d-2)-\kappa, \kappa\right)$ in its closure. 
Proof. Through a point $E$ in $V\left(d, \frac{1}{2}(d-1)(d-2)-\kappa, \kappa\right)$, there exist

$$
\left(\begin{array}{c}
\frac{1}{2}(d-1)(d-2)-\kappa \\
\frac{1}{2}(d-1)(d-2)-\kappa-\delta
\end{array}\right)
$$

sheets of the closure of $V(d, \delta, \kappa)$ each of which corresponds to smoothing some $\frac{1}{2}(d-1)(d-2)-\kappa-\delta$ nodes. Since $V\left(d, \frac{1}{2}(d-1)(d-2)-\kappa, \kappa\right)$ is irreducible, it is enough to show that these sheets come from the same component of $V(d, \delta, \kappa)$, which is equivalent to showing that we can interchange the $\frac{1}{2}(d-1)(d-2)-\kappa-\delta$ nodes as $E$ varies along a closed arc in $V\left(d, \frac{1}{2}(d-1)(d-2)-\kappa, \kappa\right)$.

For the rest of the proof we are continuing with the previous set up of (2.1). Say that $E=\pi_{\Lambda}(C)$. Note that the nodes and the cusps of $E$ correspond to the intersection points of $\Delta$ with secant lines and tangent lines of $C$, respectively.

Let $\left\{x_{1}, \ldots, x_{\kappa}\right\}=\Lambda \cap T C$, where $T C$ is the tangent variety of $C$. Note that $x_{i} \notin C$. Define $\pi_{S_{\kappa}}: \mathbf{P}^{d} \rightarrow \mathbf{P}^{d-\kappa}$ as before. We then claim that the image $C_{\kappa}$ of $C$ in $\mathbf{P}^{d-\kappa}$ is a nondegenerate rational curve in $\mathbf{P}^{d-\kappa}$ of degree $d$ with $\kappa$ cusps as its only singularities. It is easy to see that it has no more cusps since $\Lambda$ meets only $\kappa$ distinct tangent lines of $C$ transversely. Now suppose that $C_{\kappa}$ has (at best) a node. Then the $(\kappa-1)$-plane $S_{\kappa}$ would meet a secant line of $C$ besides $\kappa$ tangent lines of $C$, which is impossible since $S_{\kappa}$ with the $\kappa$ tangent lines and the secant line above will span at most $(2 \kappa)$-plane. This means that there exists a $(2 \kappa)$-plane, if $2 \kappa \leq d-1$, meeting a rational normal curve $C$ at $2 \kappa+2$ points, which is a contradiction.

Since no secant lines and tangent lines other than $t_{i}$ meet $S_{\kappa}$, they map via $\pi_{S_{K}}$ to the corresponding lines of $C_{\kappa}$. And $\pi_{S_{K}}(\Lambda)$ meets $\frac{1}{2}(d-1)(d-2)-\kappa$ distinct secant lines of $C_{\kappa}$ away from $C_{\kappa}$ since $E=\pi_{\Lambda_{\kappa}} \cdot \pi_{S_{\kappa}}(C), \Lambda_{\kappa}=\pi_{S_{\kappa}}(\Lambda)$. Let $\mathscr{C}(C)$ and $\mathscr{C}\left(C_{\kappa}\right)$ be the chodal varieties of $C$ in $\mathbf{P}^{d}$ and $C_{\kappa}$ in $\mathbf{P}^{d-\kappa}$, respectively. Suppose now that $\pi=\pi_{S_{\kappa} \mid r(C)}: \mathscr{C}(C) \rightarrow \mathscr{C}\left(C_{\kappa}\right)$ is generically one to one under our assumption $\kappa \leq \min \left(d-4, \frac{1}{2}(d-1)\right)$. Now $\{$ hyperplanes of $\mathbf{P}^{d}$ containing $\left.S_{\kappa}\right\}=\left\{\right.$ hyperplanes of $\left.\mathbf{P}^{d-\kappa}\right\}$. Thus the degree of $\mathscr{C}\left(C_{\kappa}\right)$ is equal to $\frac{1}{2}(d-1)(d-2)-\kappa$, the degree of $\mathscr{C}(C)-\kappa$, since $\mathscr{C}\left(C_{\kappa}\right)$ misses $\kappa$ points on $\mathscr{C}(C)$ under the projection $\pi_{S_{\kappa}}$. As the nodes of $E$ correspond to the intersection of $\Lambda$ and the secant lines of $C$, they correspond to the intersection of $\Lambda_{\kappa}$ and the secant lines of $C_{\kappa}$. Then applying the uniform position theorem [ACGH, H3] to $\mathscr{C}\left(C_{\kappa}\right)$ we get that the monodromy action on the points of intersection of (smooth locus) of $\mathscr{C}\left(C_{\kappa}\right)$ with a general $(d-\kappa-3)$ plane $P$ in $\mathbf{P}^{d-\kappa}$ is the full symmetric group. But a $(d-\kappa-3)$-plane $P$ in $\mathbf{P}^{d-\kappa}$ with $S_{\kappa}$ spans a $(d-3)$-plane $\Lambda$ the projection $\pi_{\Lambda}$ of $C$ from which gives a curve in $V\left(d, \frac{1}{2}(d-1)(d-2)-\kappa, \kappa\right)$. So we may assume, in the beginning of this proof, that $E=\pi_{\Lambda}(C)$ where $\Lambda$ occurs in the above way. So we are done once we show that $\pi: \mathscr{C}(C) \rightarrow \mathscr{E}\left(C_{\kappa}\right)$ is generically one to one.

Suppose not. Then, for a general point $q$ of a general secant line $L$ of $\mathscr{E}\left(C_{\kappa}\right)$, there is another secant line through $q$. Now take $L_{1}$ and $L_{2}$ two 
general secant lines meeting at $q \notin C_{\kappa}$. Let $X_{1}=\left\{p \in C_{\kappa}\right.$ : some secant line $\left(\neq L_{1}, L_{2}\right)$ through $p$ meets $\left.L_{1} \backslash q\right\}$; and $X_{2}=\left\{p \in C_{\kappa}\right.$ : some secant line $\left(\neq L_{1}, L_{2}\right)$ through $p$ meets $\left.L_{2} \backslash q\right\}$. Then both $X_{1}$ and $X_{2}$ are dense in $C_{\kappa}$, so we can choose $p \in C_{\kappa}$ whose two second lines meet $L_{1}$ and $L_{2}$ away from $C_{\kappa}$, so we can choose $p \in C_{\kappa}$ whose two secant lines meet $L_{1}$ and $L_{2}$ away from $C_{\kappa}$ and $q$. Consider the 3-plane $P_{3}$ spanned by $L_{1}, L_{2}$ and $p$. Then $P_{3}$ meets $C_{\kappa}$ at seven points. If $P_{3}$ is a hyperplane of $\mathbf{P}^{d-\kappa}$ we stop here. If not, we do the same thing and find a point $p^{\prime} \in C_{\kappa}$ at which two secant lines of $C_{\kappa}$ meet $L_{1}$ and $L_{2}$, respectively, away from $C_{\kappa}, q$ and those intersection points of $L_{i}$ with the lines we get in the previous steps to get a $(2+m)$-plane of $\mathbf{P}^{d-\kappa}$ meeting $C_{\kappa}$ at $4+3 m$ points until the $(2+m)$-plane becomes a hyperplane of $\mathbf{P}^{d-\kappa}$, i.e., $m=d-\kappa-3$ and $4+3 m=3 d-3 \kappa-5$. Since $\operatorname{deg}\left(C_{\kappa}\right)=d, \pi$ cannot be multiple to one if $3 d-3 \kappa-5>d$, equivalently, if $\kappa<(2 d-5) / 3$. Note that $\kappa<(2 d-5) / 3$ if $\kappa \leq \min \left(d-4, \frac{1}{2}(d-1)\right)$ except when $d=7$ and $\kappa=3$. If $d=7, \kappa=3$ and $\pi$ is multiple to one, then we cannot have in $\mathbf{P}^{3}$ a rational curve of degree 7 with 3 cusps and one node because any such curve in $\mathbf{P}^{3}$ would be a projection of $C_{\kappa}=C_{3} \subset \mathbf{P}^{4}$ from a point $q$ on some general secant line of $C_{3}$ and there are at least two secant lines through $q$. But now consider a rational plane curve $D$ of degree 5 with three cusps and three nodes, blow up $\mathbf{P}^{2}$ at two nodes and four smooth points of $D$ (no three collinear nor all on a conic), and then embed it in $\mathbf{P}^{3}$ via the system of cubics through the 6 blown up points to get a rational curve of degree 7 in $\mathbf{P}^{3}$ with three cusps and one node. Therefore $\pi: \mathscr{C}(C) \rightarrow \mathscr{C}\left(C_{\kappa}\right)$ is generically one to one.

(2.3) Remark. Even though (2.1) or (2.2) does not include some cases of Theorem (1.2) when $d=4$ and $d=5$, we can see that they are still valid.

(1) If $d=4$ and $\kappa=3$, then $V(4,0,3)$ is the only possibility and it is irreducible because it is the family of duals of elements of $V(3,1)$, the locus of reduced and irreducible plane curves of degree 3 having one node as their only singular point.

(2) If $d-\kappa=3$ in the proof of (2.2) then $\mathscr{C}\left(C_{\kappa}\right)$ is $\mathbf{P}^{3}$ or dense in $\mathbf{P}^{3}$ and any $(d-3)$-plane in $\bigcap_{i=1}^{\kappa} \sigma_{3}\left(x_{i}\right)$ maps to a point. So we cannot use the uniform position theorem as we did in (2.2). But if $d=4$ and $\kappa=1$, a $(d-3)$-plane $\Lambda$ which is a line in $\mathbf{P}^{4}$ maps to a point in $\mathbf{P}^{3}$ through which two secant lines pass. Now we have a two-fold covering from $\mathscr{C}(C)$ to (a dense set) of $\mathbf{P}^{3}$. Considering the monodromy action to this map we can show that the monodromy group is $S_{2}$, the full symmetric group on two elements.

If $d=5$ and $\kappa=2$, then any $\Lambda \in \bigcap_{i=1}^{\kappa} \sigma_{3}\left(x_{i}\right)$ maps via $\pi_{S_{\kappa}}$ to a point in $\mathbf{P}^{3}$ at which four secant lines meet. So we have a four-fold covering from $\mathscr{C}(C)$ to a (dense set of) $\mathbf{P}^{3}$. If $d=5$ and $\kappa=2$ then $V(5,4,2)$ which consists of rational curves, $V(5,3,2), V(5,2,2), V(5,1,2)$ and $V(5,0,2)$ are the only possibilities. Considering the monodromy action to the four-fold 
covering we can show that $(2.2)$ remains true for $V(5,3,2)$ and $V(5,1,2)$. To get $V(5,0,2)$ from $V(5,4,2)$ we have to smooth all four nodes, so there exists only one branch of $V(5,0,2)$ through each member of $V(5,4,2)$. For $V(5,2,2)$, we prove it by direct computation: first note on three singular points of degree 5 curve are collinear; pick four points $(1,0,0),(0,1,0),(0,0,1)$ and $(1,1,1)$, first two for cusps and the others for nodes, and show by explicitly writing down equations that the family of curves in $V(5,2,2)$ which have their nodes and cusps fixed as above is irreducible. Since we can always move by automorphisms of $\mathbf{P}^{2}$ four general points to the above four points, it is done.

(3) If $d=4$ and $\kappa=2$, there is again only one sheet of $V(4,0,2)$ in its closure through each general member of $V(4,1,2)$.

Let $U(d, \delta, \kappa)$ be the locus of points of $\mathbf{P}^{N}$ corresponding to reduced plane curves of degree $d$ with $\delta$ nodes and $\kappa$ cusps as their only singularities. In this section we want to estimate the dimensions of some subloci of $V(d, \delta, \kappa)$ or $U(d, \delta, \kappa)$ which we will use in the next section. We begin this by introducing a slightly larger locus than $U(d, \delta, \kappa)$. Let $U^{d, g, c}\left(V^{d, g, c}\right.$ resp.) in $\mathbf{P}^{N}$ be the locus consisting of reduced (and irreducible resp.) plane curves of degree $d$, genus $g$ and class $c$. Then $V^{d, g, c}$ is a union of some components of $U^{d, g, c}$ whose general member is irreducible. Diaz and Harris then showed

(3.1) Theorem [DH1, (1.2)]. If $c \geq 2 g-1$, then any component $W$ of $V^{d, g, c}$ has dimension $d+c-g+1$ and any singular point of a general member $C$ of $V^{d, g, c}$ is either a node or a cusp. In fact, $C$ has $\delta$ nodes and $\kappa$ cusps where $g=\frac{1}{2}(d-1)(d-2)=\delta-\kappa$ and $c=d(d-1)-2 \delta-3 \kappa$.

Unless confusion arises we mean $g=\frac{1}{2}(d-1)(d-2)-\delta-\kappa$ and $c=$ $d(d-1)=2 \delta-3 \kappa$ in the context of $U^{d, g, c}$ or $U(d, \delta, \kappa)$. Note that the condition $c \geq 2 g-1$ in (3.1) can be read as $\kappa \leq 2 d-1$ and $d+c-g+1$ as $g+3 d-\kappa-1$. By examining Theorem (3.1) for each $d$ one by one we get

(3.2) Corollary. Let $\kappa \leq 11$. If $W$ is a component of $U^{d, g, c}$, then $\operatorname{dim} W=$ $d+c-g+1$ and the general member of $W$ has $\delta$ nodes and $\kappa$ cusps as its only singularities.

Throughout this section the main tools are the following propositions.

(3.3) Proposition [H1]. Let $S$ be a smooth rational surface, $D$ a divisor class on $S$, and $W \subset|D|$ a family of reduced curves of geometric genus $g$ in the linear system $|D|$. Assume that for a general member $E \in W$ the restriction to each component of $E$ of the anti-canonical divisor class $-K_{S}$ contains an effective divisor of positive degree. Then

(1) $\operatorname{dim} W \leq g-D \cdot K_{S}-1$.

(2) If $\operatorname{dim} W=g-D \cdot K_{S}-1$, then the general member of $W$ has only nodes as its singularities; and 
(3) if $\operatorname{dim} W=g-D \cdot K_{S}-1$ and $C \subset S$ is any curve, the general member of $W$ meets $C$ transversely.

Note that if $\eta: X \rightarrow \mathbf{P}^{2}$ is a map, by $\eta(X)$ we will not mean the reduced image, but rather the image with multiplicities, so that $\operatorname{deg} \eta(X)=\operatorname{deg}\left(\eta^{*} \mathscr{O}_{\mathbf{P}^{2}}(1)\right)$.

(3.4) Proposition [H1]. Let $S$ be a smooth rational surface. Let $\pi: \Xi \rightarrow B$ be a family of curves of geometric genus $g$ with $B$ irreducible, and $\eta: \Xi \rightarrow S$ a map such that if $X_{b}=\pi^{-1}(b)$ is a general fiber, $\eta$ is not constant on any component $Z$ of $X_{b}$, and the restriction of $-K_{S}$ to the reduced image $Z^{\prime}$ of $Z$ is linearly equivalent on $Z^{\prime}$ to a positive effective divisor, and let $W$ be the locus of images $\left\{\eta\left(X_{b}\right)\right\}_{b \in B}$ of fibers of $\pi$ under the map. Then

(1) $\operatorname{dim} W \leq g-\operatorname{deg}\left(\left.\eta^{*} K_{S}\right|_{X_{b}}\right)-1$; and

(2) if equality holds in (1) then the map $\eta \mid X_{b}$ is birational onto its image, and the conlusions of parts (2) and (3) of Proposition (3.3) above apply to $\eta\left(X_{b}\right)$ accordingly.

(3.5) Definition. Let $C$ be a reduced curve on a nonsingular surface $S, q$ a singular point of $C$. Take a sufficiently small neighborhood $N$ of $q$ so that, if $h: C^{\prime} \rightarrow C$ is the normalization of $C$ over $N$ and $C_{1}^{\prime}, C_{2}^{\prime}, \ldots, C_{n}^{\prime}$ are the components of $C^{\prime}$, each $C_{i}^{\prime}$ contains only one preimage of $q$. Define then

$$
\bar{m}_{q}(C)=\sum_{i}\left[\operatorname{mult}_{q} h\left(C_{i}^{\prime}\right)-1\right] \text { and } \bar{m}(C)=\sum_{q \in C} \bar{m}_{q}(C) \text {. }
$$

If $C$ has only one singular branch at $q$ then $\bar{m}_{q}(C) \geq 1$, and with equality if and only if the singular branch of $C$ is locally given by $y^{2}=x^{2 n+1}$ for some positive integer $n$.

If $W$ is an equigeneric family of reduced curves on a nonsingular surface $S$ and has $\bar{m}(C)$ constant for all $C \in W$ then we call $W$ an equiclassical family. (Cf. [DH1] for details, in particular for the case $S=\mathbf{P}^{2}$.)

(3.6) Proposition. Let $S$ be a smooth surface, $D$ a divisor class, and $W \subset|D|$ an equiclassical family of reduced and irreducible curves $C$ in $S$ of genus $g$ and $\bar{m}(C)=\kappa$. Then

$$
\operatorname{dim} W \leq \max \left(g-K_{S} \cdot D-\kappa-1, g\right) .
$$

Proof. Zariski [Z2] established the following fact: let $W \subset|D|$ be a family of curves in a smooth surface, then the tangent space $T_{C} W$ to $W$ at a general point $C \in W$ be identified as a subspace of $H^{0}\left(\mathscr{O}_{C}(C) \otimes I\right)$, where $I$ is the equisingular ideal of $C$. Let $H$ be the equiclassical ideal of $C$ (see [DH1] for details) then $I \subset H$. Thus

$$
\operatorname{dim} W \leq h^{0}\left(\mathscr{O}_{C}(C) \otimes I\right) \leq h^{0}\left(\mathscr{O}_{C}(C) \otimes H\right) .
$$

Let $\varphi: C^{\prime} \rightarrow C$ be the normalization of $C$. Then there exist two divisors $A^{\prime}$ and $R^{\prime}$ on $C^{\prime}$ of degrees $2\left(p_{a}(C)-p_{g}(C)\right)$ twice the difference between the 
arithmetic and the geometric genera of $C$ and $\bar{m}(C)=\kappa$ respectively, so that there is an inclusion map

$$
H^{0}\left(\mathscr{C}_{C}(C) \otimes H\right) \hookrightarrow H^{0}\left(C^{\prime}, \varphi^{*} \mathscr{O}_{C}(C)-A^{\prime}-R^{\prime}\right) \quad(\mathrm{cf} .[\mathrm{DH} 1]) .
$$

Note that $\operatorname{deg}\left(\varphi^{*} \mathscr{O}_{C}(C)-A^{\prime}-R^{\prime}\right)=C \cdot C-2\left\{\frac{1}{2}\left(C \cdot C+K_{S} \cdot C\right)+1-g\right\}-\kappa=$ $-K_{S} \cdot C-2+2 g-\kappa$. If $-K_{S} \cdot C \geq \kappa+1$ then $\varphi^{*} \mathscr{O}_{C}(C)-A^{\prime}-R^{\prime}$ is nonspecial. Applying the Riemann-Roch theorem to $\varphi^{*} \mathscr{O}_{C}(C)-A^{\prime}-R^{\prime}$ on $C^{\prime}$ we have $\operatorname{dim} W \leq \operatorname{deg}\left(\varphi^{*} \mathscr{O}_{C}(C)-A^{\prime}-R^{\prime}\right)-g+1=-K_{S} \cdot C-2+2 g-\kappa-g+1=$ $g-K_{S} \cdot C-\kappa-1$. If $-K_{S} \cdot C \leq \kappa=\bar{m}(C)$, then $\operatorname{deg}\left(\varphi^{*} \mathscr{O}_{C}(C)-A^{\prime}-R^{\prime}\right) \leq 2 g-2$ and, by Riemann-Roch,

$$
\operatorname{dim} W \leq \operatorname{deg}\left(\varphi^{*} \mathscr{O}_{C}(C)-A^{\prime}-R^{\prime}\right)-g+1+h^{1}\left(\varphi^{*} \mathscr{O}_{C}(C)-A^{\prime}-R^{\prime}\right) \leq g,
$$

since

$$
\begin{aligned}
h^{1}\left(\varphi^{*} \mathscr{O}_{C}(C)-A^{\prime}-R^{\prime}\right) & =h^{0}\left(K_{C^{\prime}}-\varphi^{*} \mathscr{O}_{C}(C)+A^{\prime}+R^{\prime}\right) \\
& \leq \operatorname{deg}\left(K_{C^{\prime}}-\varphi^{*} \mathscr{\mathscr { O }}_{C}(C)+A^{\prime}+R^{\prime}\right)+1 .
\end{aligned}
$$

Let $U^{d, g}\left(V^{d, g}\right.$ resp.) in $\mathbf{P}^{N}$ be the closure of the locus of reduced (and irreducible resp.) plane curves of degree $d$ and genus $g$. Unlike the degeneration of $V^{d, g}$ to $V^{d, 0}$ in [H1] we have to control cusps too. Therefore we consider here various subloci of $U(d, \delta, \kappa)$ or $U^{d, g, c}$ defined by requiring that for a general member the cusps lie at certain fixed points. To begin with we choose and then fix $\kappa$ distinct points $Q_{1}, \ldots, Q_{\kappa}$ no three or which are collinear and a line $L$ missing them. Now define

(3.7) $U_{m, \kappa}^{d, g}\left(V_{m, \kappa}^{d, g}\right.$ resp.) as the closure of the locus of all reduced (and irreducible resp.) plane curves $C$ of degree $d$ and genus $g$ which do not contain $L$, have a contact of order $m$ with $L$ at a point $p$ (a smooth point $p$ resp.) of $C$, and satisfy $\bar{m}_{Q_{i}}(C) \geq 1$ at the fixed $\kappa$ points $Q_{i}$.

For the rest of this paper $\kappa \leq 3$, and $\kappa$ points $Q_{i}$ and $L$ are fixed unless otherwise specified.

(3.8) Lemma. Let $X$ be a component of $U_{m, \kappa}^{d, g}$. For $\kappa \leq 3$ we have

(1) if $X$ is nonempty, then

(a) $\operatorname{dim} X=g+3 d-3 \kappa-m$; and

(b) if $C$ is a general point of $X,(m \geq 2)$ the curve $C$ has a unique point $p$ of intersection multiplicity $m$ with $L$; in a neighborhood of $p, C$ is a union of smooth arcs $C^{1}, \ldots, C^{k}$ having contact of orders $a_{1}, \ldots, a_{k}$ with $L$ respectively, $\sum a_{i}=m$, having minimal order of contact among themselves:

$$
\left(C^{i} \cdot C^{j}\right)_{p}=\min \left(a_{i}, a_{j}\right),
$$

and $(m \geq 1) \quad C$ has cusps at $Q_{i}$ and the remaining singularities of $C$ are all nodes. 
(2) Let $\pi: \Xi \rightarrow B$ be a family of smooth curves of genus $g$ with $B$ irreducible, $\eta: \Xi \rightarrow \mathbf{P}^{2}$ a map whose restriction to a general fiber $X_{b}$ of $\Xi$ has degree $d$, is not constant on any component of $X_{b}$, does not map any component of $X_{b}$ to $L, \eta\left(X_{b}\right)$ has a singular branch at each $Q_{i}$ and has contact of order $m$ with $L$ at some point $p \in L$. Let $X=\left\{\eta\left(X_{b}\right)\right\}_{b \in B} \subset$ $\mathbf{P}^{N}$ be the locus of images. Then $\operatorname{dim}(X) \leq g+3 d-3 \kappa-m$, and if $\operatorname{dim}(X)=g+3 d-3 \kappa-m, \eta \mid X_{b}$ is birational and $X$ is dense in a component of $U_{m, \kappa}^{d, g}$.

Proof. We begin the proof by introducing the following: for a component $W$ of $U^{d, g, c}$, let

$W_{m}=$ (the closure in $U^{d, g}$ of) $\{C \in W \mid C$ does not contain $L$ and $(C \cdot L)_{p}=m$ for some point $\left.p \in L\right\}$; and

$W_{m, \kappa}=$ (the closure in $U^{d, g}$ of) $\left\{C \in W \mid C\right.$ satisfies $\bar{m}_{Q_{i}}(C) \geq 1$, does not contain $L$ and $(C \cdot L)_{p}=m$ for some point $\left.p \in L\right\}$.

We first consider a component $X$ of $U_{m, \kappa}^{d, g}$ whose general member $C$ is irreducible. Suppose $\bar{m}(C)=\kappa^{\prime} \geq \kappa$. Then we may assume $X \subset V^{d, g, c^{\prime}}$ where $c^{\prime}=2(d-1)+2 g-\kappa^{\prime}$. Let $W^{\prime}$ be a component of $V^{d, g, c^{\prime}}$. Fix now a point $p \in L$ and let $W_{m}^{\prime 0}$ be the locus of curves $C$ in $W_{m}^{\prime}$ having intersection number $m$ with $L$ at $p$ specifically but no cusps in general on $L$. Since $\operatorname{dim} V^{d, g, c^{\prime}}=g+3 d-\kappa^{\prime}-1$ and $(L \cdot C)_{p}=m$ is expressed by $m$ equations on the coefficients of $C$, we have $\operatorname{dim} W_{m}^{\prime 0} \geq g+3 d-\kappa^{\prime}-m-1$ if nonempty.

To prove that $\operatorname{dim} W_{m}^{\prime 0} \leq g+3 d-\kappa^{\prime}-m-1$, we blow up $\mathbf{P}^{2}$ at $p m$ times in the direction of $L$ : let $S_{1}$ be the blow-up of $\mathbf{P}^{2}$ at $p, E_{1}$ the exceptional divisor, and $p_{1} \in E_{1}$ the point corresponding to $L$; define blow-ups $S_{i}$ by letting $S_{i+1}$ be the blow-up of $S_{i}$ at $p_{i}, E_{i+1}$ the exceptional divisor, and $p_{i+1} \in E_{i+1}$ the point of intersection of $E_{i+1}$ with the proper transform of $L$. We blow up exactly $m$ times if $p$ is a smooth point of $C$; otherwise we may stop before. Let $C$ be a general member of $W_{m}^{\prime 0}, C_{i}$ the proper transform of $C$ in $S_{i}$, and $b_{i+1}$ the multiplicity of $C_{i}$ at $p_{i}$. Letting $\pi_{i}: S_{m} \rightarrow S_{i}$ and $\pi: S_{m} \rightarrow \mathbf{P}^{2}$ we have on $S_{m}$ that $C_{m} \sim \pi^{*} C-\sum_{i}^{m} b_{i} \pi_{i}^{*} E_{i}$ with $\sum_{i}^{m} b_{i}=m$ and

$$
K_{S_{m}}=\pi^{*}(-3 H)+\sum_{i}^{m} \pi_{i}^{*} E_{i}
$$

where $H$ is a hyperplane divisor of $\mathbf{P}^{2}$. Therefore

$$
-K_{S_{m}} \cdot C_{m}=3 d-m
$$

which is at least $2 d$ (when $m=d$ ). Applying (3.6) to the family $\left\{C_{m} \mid C \in\right.$ $\left.W_{m}^{\prime 0}\right\}$ of curves in the above, we get

$$
\operatorname{dim} W_{m}^{\prime 0} \leq g+3 d-m-\kappa^{\prime}-1
$$


and

$$
\operatorname{dim} W_{m}^{\prime} \leq g+3 d-m-\kappa^{\prime}
$$

(in fact an equality). Since there is an automorphism of $\mathbf{P}^{2}$ which maps the line $L$ to itself and three general points to another three general points,

$$
\operatorname{dim} X \leq g+3 d-m-\kappa^{\prime}-2 \kappa \leq g+3 d-m-3 \kappa
$$

with equality only if $\kappa^{\prime}=\kappa$, consequently only if $\bar{m}_{Q_{i}}(C)=1$.

For (1b), therefore, we may assume that $X$ is a component of $W_{m, \kappa}$ where $W$ is a component of $V^{d, g, c}$ with $c=2(d-1)+2 g-\kappa$. Repeating the previous dimension count by replacing $W^{\prime}$ by $W$ and $\kappa^{\prime}$ by $\kappa$, we have

$$
\operatorname{dim} W_{m, \kappa}=g+3 d-3 \kappa-m .
$$

We can also see, from the dimension counts in the previous paragraph, that if $C$ is a general member of $W_{m, \kappa}$, then $C$ is again a general one of $W_{m}^{0}$. Thus we can easily remove the possibility that $\bar{m}\left(C_{m}\right)>\kappa$. Since we require that $\bar{m}_{Q_{i}}(C) \geq 1$ for all $i, C$ satisfies $\bar{m}_{Q_{i}}(C)=1$ and has only one singular branch $C_{i}$ at each $Q_{i}$ possibly with smooth branches through each $Q_{i}$. Now suppose that a general curve $C$ in $W_{m, \kappa}$ has a more complicated singular point than a cusp, say, at $Q_{1}$. To count the dimension of a family of such curves $C$ we fix a line $T_{1}$ through $Q_{1}$ as a tangent line of a singular branch of $C$ and assume that $C$ has, locally at $Q_{1}, r$ smooth branches transverse to $T_{1}$ and $s$ smooth branches tangent to $T_{1}$ as well as one singular branch $C_{s}$. Let $x$ and $y$ be a local coordinate of $\mathbf{P}^{2}$ at $q_{1}$ and $T_{1}$ be $\{y=0\}$. Then we blow up $S_{m}$ as follows:

(3.8.1) Let $S_{m+1}$ be the blow-up of $S_{m}$ at $Q_{1}, E_{m+1}$ the exceptional divisor and $Q_{1}^{1} \in E_{m+1}$ the point corresponding to $T_{1}$; if a curve $C$ in $\mathbf{P}^{2}$ has an ordinary cusp at $Q_{1} \quad\left(y^{2}=x^{3}\right)$, then its proper transform $C_{m+1}$ in $S_{m+1}$ meets $E_{m+1}$ with multiplicity $\left(E_{m+1} \cdot C_{m+1}\right)_{Q_{1}^{1}}=2$ at a smooth point $Q_{1}^{1}$ of $C_{m+1}$; blowing up $S_{m+1}$ at $Q_{1}^{1}$ (call it $S_{m+2}$ ) and again $S_{m+2}$ at $Q_{1}^{2}$ the point corresponding to $E_{m+1}$ on $S_{m+2}$ to get $S_{m+3}$. Then on $S_{m+3}$, if a singular branch of a general curve $C$ has a cusp at $Q_{1}$, the proper transforms $C_{m+3}$ of $C$ in $W_{m}^{0}$ with only $Q_{1}$ and $T_{1}$ fixed form an equiclassical family of curves of genus $g$ with $\bar{m}\left(C_{m+3}\right)=\kappa-1$. Let $\pi_{i}: S_{m+3} \rightarrow S_{i}$ and $E_{i}$ the exceptional curve of $S_{i} \rightarrow S_{i-1}$ for $1 \leq i \leq m+3$, here $S_{0}=\mathbf{P}^{2}$. Then

$$
C_{m+3} \simeq \pi^{*} C-\sum_{i}^{m} b_{i} \pi_{i}^{*} E_{i}-(r+s+2) \pi_{m+1}^{*} E_{m+1}-(s+1) \pi_{m+2}^{*} E_{m+2}-E_{m+3}
$$

where $\sum_{i}^{m} b_{i}=m$. So $-K_{m+3} \cdot C_{m+3}=3 d-m-r-2 s-4$. (From now on $K_{i}$ is the canonical divisor of $S_{i}$.) If the local equation of a singular branch is given 
by $y^{2}=x^{2 n+1}, n \geq 2$, then the proper transform $C_{m+3}$ of $C$ is equivalent to

$$
\pi^{*} C-\sum_{i}^{m} \pi_{i}^{*} E_{i}-(r+s+2) \pi_{m+1}^{*} E_{m+1}-(s+2) \pi_{m+2}^{*} E_{m+2}
$$

with $\bar{m}\left(C_{m+3}\right)=\kappa-1$ provided that $n=2$ or $\bar{m}\left(C_{m+3}\right)=\kappa$ otherwise. In either case, $-\kappa_{m+3} \cdot C_{m+3}=3 d-m-r-2 s-4$ which is big enough to apply (3.6) since $r+2 s+2 n+1 \leq d$ by considering the intersection multiplicity of $T_{1}$ and $C$ at $Q_{1}$. Thus $\operatorname{dim}\left\{C \in W_{m}^{0} \mid\right.$ with $Q_{1}$ and $T_{1}$ fixed $\} \leq g+$ $3 d-m-r-2 s-4-\kappa$ if $n=1$ or 2 ; or $\operatorname{dim}\left\{C \in W_{m}^{0} \mid\right.$ with $Q_{1}$ and $T_{1}$ fixed $\} \leq g+3 d-m-r-2 s-5-\kappa$ if $n \geq 3$. Removing the restriction on $T_{1}$ and moving $Q_{1}$ to any point we want, $\operatorname{dim} W_{m}^{0} \leq g+3 d-m-r-2 s-\kappa-1$ if $n=1$ or 2 ; or $\operatorname{dim} W_{m}^{0} \leq g+3 d-m-r-2 s-\kappa-2$ if $n \geq 3$, which is always less than $g+3 d-m-\kappa-1$ the actual dimension of $W_{m}^{0}$ provided either that $r$ or $s$ is not zero, or that $n \geq 3$.

Now suppose that the local equation of $C$ at $Q_{1}$ is $y^{2}=x^{5}$. Then we blow up $S_{m}$ as follows: let $S_{m+1}$ be the blow-up of $S_{m}$ at $Q_{1}, E_{m+1}$ the exceptional curve, $Q_{1}^{1}$ the point corresponding to $T_{1}, C_{m+1}$ the proper transform of $C$ in $S_{m+1}$; then $C_{m+1}$ has an ordinary cusp at $Q_{1}^{1}$; now blow up $S_{m+1}$ beginning at $Q_{1}^{1}$ three times as we did in (3.8.1) to resolve a cusp, call $S_{m+4}$ the final blown up surface. On $S_{m+4},\left\{C_{m+4} \mid C \in W_{m}^{0}\right.$ with $Q_{1}$ and $T_{1}$ fixed $\}$ forms an equiclassical family of genus $g$ and $\bar{m}\left(C_{m+4}\right)=\kappa-1$. Again by (3.6), since $-K_{m+4} \cdot C_{m+4}=3 d-6-m$ where $K_{m+4}$ is the canonical divisor of $S_{m+4}$, this family has dimension at most $g+(3 d-6-m)-(\kappa-1)-1$, therefore $\operatorname{dim} W_{m}^{0} \leq g+3 d-\kappa-m-3$, which again gives a contradiction.

Suppose this time that a general member $C$ of $W_{m, \kappa}$ has a singular point other than a node away from $Q_{i}$ and $L$. Then we consider the sublocus of $W_{m}^{0}$ of curves $C$ through a fixed point $q$ (not $p$ nor in $L$ ) which is a singular point with $\bar{m}_{q}(C)=0$ but not a node of $C$, so either all branches at $q$ are transverse or some are not. By blowing up $S_{m}$ at $q$ (once if all branches are transverse, twice if some are tangential; see (3.10)) and counting dimension of each component in a similar way we get a contradiction again since the dimension of $W_{m}^{0}$ goes down at least by 1 .

We now claim that if $C$ is a general member of $W_{m}^{0}$ then $C_{m}$ in $S_{m}$ must meet the union $E=\bigcup \widetilde{E}_{i}$ of the $\widetilde{E}_{i}$ transversely: otherwise we blow up those points (including the intersections $\widetilde{E}_{i} \cap \widetilde{E}_{j}$ ) at which $C_{m}$ and $E$ do not meet transversely and apply another dimension count to get a contradiction. In particular, $C_{m}$ will meet each $\widetilde{E}_{i}$ transversely at $b_{i}-b_{i+1}$ distinct points other than $\widetilde{E}_{i} \cap \widetilde{E}_{i-1}$ or $\widetilde{E}_{i} \cap \widetilde{E}_{i+1}$. Then a branch $C^{i}$ of $C$ whose proper transform in $C_{m}$ crosses $\widetilde{E}_{a_{i}}$ will then be smooth and have contact of order $a_{i}$ with $L$ at $p$; two branches $C^{i}, C^{j}$, since their proper transforms cross $E$ at different points, will have contact of order $\min \left(a_{i}, a_{j}\right)$. 
If a general curve $C$ has a point $q \neq p$ in $L$ of intersection multiplicity bigger than 1 with $L$ then, by blowing up $S_{m}$ at $q$ as we did for $p$, we get a contradiction as well.

Let $W_{m, \kappa}^{0}=\left\{C \in W_{m, \kappa} \mid(C \cdot L)_{p}=m\right.$ for a fixed point $p$ in $\left.L\right\}$. Then if $\kappa \leq 2, \operatorname{dim} W_{m, \kappa}^{0}=g+3 d-m-3 \kappa-1$ since there is an automorphism fixing two points $Q_{1}$ and $Q_{2}$, and mapping $p$ to any point in $L$ except $L \cap L^{\prime}$, where $L^{\prime}$ is the line through $Q_{1}$ and $Q_{2}$.

To prove the lemma in case that $C$ is reducible, suppose that $C$ has $k$ components $C_{i}$ of genus $g_{i}$ and degree $d_{i}$ with a contact of multiplicity $m_{i}$ with $L$ at a fixed point $p$ in $L$. If $\kappa=1$ there is an easy way to see the proof of lemma; we may blow up $S_{m}$ at $Q_{1}$ three times as in (3.8.1); then on $S_{m+3}-K_{m+3}$ the anticanonical divisor is equivalent to an effective divisor and any irreducible curve in $S_{m+3}$ other than the proper transform $\widetilde{L}$ of $L$ or $\widetilde{E}_{i}$ of $E_{i}$ meets $-K_{m+3}$ positively; applying now Propositions (3.3) and (3.4) to a family of curves in $S_{m+3}$ in the divisor class $\mid \pi^{*} d H-\sum_{i}^{m} b_{i} \pi_{i}^{*} E_{i}-$ $2 \pi_{m+1}^{*} E_{m+1}-\pi_{m+2}^{*} E_{m+2}-E_{m+3} \mid$ we have part (1) and (2).

If $\kappa=2$ we may assume either that $C_{1}$ has two cusps and the other components have no cusps, or that each $C_{i}$ has a cusp at $Q_{i}$ for $i=1,2$ and the others have no cusps at all. Then if the former case happens $C_{1}$ moves in a family of dimension at most $g_{1}+3 d_{1}-m_{1}-7$ and $C_{i}$ by [H1, Lemma (2.4)] at most $g_{i}+3 d_{i}-m_{i}-1$ which is the dimension of $\left(V^{d, g}\right)_{m}^{0}$ for $i \geq 2$, therefore $C$ in a family of dimension at most $3 d+g-m-7$ since $g=\sum g_{i}-k+1$. If the latter case happens then $C_{i}$ moves in a family of dimension at most $g_{i}+3 d_{i}-m_{i}-4$ for $i=1$ and $2 ; g_{i}+3 d_{i}-m_{i}-1$ for $i \geq 3$, therefore $C$ at most $3 d+g-m-7$. If we have an equality in either case, then all $C_{i}$ must meet transversely except at $p$ the point of intersection number $m$ between $C$ and $L$ and no $C_{i}$ meet at a point in $L$ except $p$ : if not, we blow up $S_{m}$ a proper number of times at each of those possible unwanted points and apply another dimension count to get a contradiction. We also have equalities for all $C_{i}$. From the case that $C$ is irreducible and [H1, Lemma (2.4)] we have the desired results.

If $\kappa=3$, it is enough to consider the following three possibilities: (i) each $C_{i}$ has a cusp at $Q_{i}$ for $i=1,2,3$ and the other components have no cusp; (ii) $C_{1}$ has a cusp at $Q_{1}, C_{2}$ has cusps at $Q_{2}$ and $Q_{3}$ and the others have no cusps; (iii) $C_{1}$ has all three cusps and the others have no cusps at all. In (i) and (ii), the proofs are exactly the same as the case $\kappa=2$ since $\operatorname{dim} W_{m, \kappa}^{0}=$ $g+3 d-m-3 \kappa-1$ if $\kappa \leq 2$. For (iii) we know, from the case of $V_{m, k}^{d, g}$, that $C_{1}$ moves in a family of dimension at most $g_{1}+3 d_{1}-m_{1}-9$ without fixing a point $p$ in $L$ if $m_{1}$ is positive. (If $m_{1}$ is zero, it is easy to see that $C_{1}$ moves in a family of dimension at most $g_{1}+3 d_{1}-10$ and then choose $p \in L$ for the remaining $C_{i}$.) Then for each $C_{1}, p \in L$ is automatically fixed and $C_{i}$ moves in a family of dimension at most $g_{i}+3 d_{i}-m_{i}-1$ for $i \geq 2$. Therefore $C$ moves 
in a family of dimension at most $(g+k-1)+3 d-m-9-(k-1)=g+3 d-m-9$, and with equality holding only if we have equality at each stage. From the case of irreducible curves, [H1, Lemma (2.4)] and the above proof of $\kappa=2$ for transverse intersections between components of $C$, a general curve satisfies the description in (3.8) with cusps at $Q_{i}$.

Part (2) follows easily from part (1). Suppose that $X_{b}$ has components $X^{1}, \ldots, X^{k}, X^{j}$ of genus $g_{j}$, and $\left.\eta\right|_{X^{j}}$ has degree $d_{j}$, has contact of order $m_{j}$ with $L$ at $p$, and has degree $n$ over its image. Then divide the cases as before according to how many $Q_{i}$ the reduced image of each $\eta\left(X^{j}\right)$ has. Q.E.D.

This time we consider the locus $U_{m, l, \kappa}^{d, g}$ of curves $C$ in $U^{d, g}$ which do not contain $L$, satisfy $\bar{m}_{Q_{i}}(C) \geq 1$ (so $c(C) \leq c$ ) and $\sum_{i}(C \cdot L)_{p_{i}}=m$ for some collection of $l$ points $p_{1}, \ldots, p_{l}$ of $L$. Then we have

(3.9) Lemma. Let $\kappa \leq 3$. Then

(1) $\operatorname{dim} U_{m, l, \kappa}^{d, g} \leq g+3 d-3 \kappa-m+l-1$,

(2) let $\pi: \Xi \rightarrow B$ be a family of smooth curves of genus $g, \eta: \Xi \rightarrow \mathbf{P}^{2} a$ map whose restriction to a general fiber $X_{b}$ of $\pi$ has degree $d$, is not constant on any component of $X_{b}$, does not map any component of $X_{b}$ to $L$, for some set of distinct points $p_{1}, \ldots, p_{l}$ in $L$ has total order of contact

$$
\sum_{i} \sum_{q \in \eta^{-1}\left(p_{i}\right)} \operatorname{mult}_{q}\left(\eta^{*} L\right)=m
$$

and on its reduced image $C_{b}$ of $\eta\left(X_{b}\right) \quad \bar{m}_{Q_{i}}\left(C_{b}\right) \geq 1$. Let $X=\left\{\eta\left(X_{b}\right)\right\}_{b \in B} \subset$ $\mathbf{P}^{N}$ be the locus of images. Then

$$
\operatorname{dim} X \leq g+3 d-3 \kappa-m+l-1 .
$$

Proof. The proof of part (1) is very similar to that of part (1) of (3.8): we fix $l$ points $p_{1}, p_{2}, \ldots, p_{l}$ and blow up $\mathbf{P}^{2}$ at $p_{1}, \ldots, p_{l}$ at most $m$ times in the direction of $L$, i.e., blow up $\mathbf{P}^{2}$ at $p_{i}$ until the proper transform of a general curve $C$ does not meet the proper transform of $L$. Call the blown up space $S_{m}$. The family $V_{m, l}^{d, g, 0}=\left\{C \in V^{d, g} \mid \sum_{i}(C \cdot L)_{p_{i}}=m\right.$ for fixed $l$ points $p_{1}, \ldots, p_{l}$ and $C$ has $\kappa$ points (not fixed) away from $L$ at each of which $\bar{m}(C) \geq 1$ \} gives a family of curves $\bar{C}$ the proper transform of $C$ in $S_{m}$ of genus $g$ and $\bar{m}(\bar{C}) \geq \kappa$ with $\bar{C} \cdot\left(-K_{m}\right)=3 d-m$. By (3.6) $\operatorname{dim} V_{m, l}^{d, g, 0} \leq g+3 d-m-\kappa-1$, so $\operatorname{dim} V_{m, l, \kappa}^{d, g} \leq g+3 d-m-3 \kappa+l-1$.

The case of reducible curves and (2) follow from (1) just as in (3.8). Q.E.D.

(3.10) Lemma. Let $T N^{d, g}(\kappa)$ be the closure in $V^{d, g}$ of the locus of reduced and irreducible curves of degree $d$ and genus $g$ having $\kappa(\kappa \leq 3)$ tacnodes away from $L$ and satisfying

$$
\sum_{i}(C \cdot L)_{p_{i}}=m
$$


for some collection of $l$ points $p_{1}, \ldots, p_{l}$ in $L$. Then

(1) $\operatorname{dim} T N^{d, g}(\kappa) \leq g+3 d-m-\kappa+l-1$.

Let $T N_{\kappa}^{d, g}(\kappa)$ be the sublocus of $T N^{d, g}(\kappa)$ whose members have their tacnodes at the fixed $\kappa$ distinct points $Q_{i}, 1 \leq i \leq \kappa$. Since there is an (the) automorphism of $\mathbf{P}^{2}$ which maps $L$ to itself and moves three general points to another three general points of $\mathbf{P}^{2}$ we have

(2) $\operatorname{dim} T N_{\kappa}^{d, g}(\kappa) \leq g+3 d-m-3 \kappa+l-1$.

We mean by a tacnode a point where a curve $C$ has two smooth branches meeting tangentially.

Proof. Let $\kappa=1$. Fixing then $l$ specific points $p_{1}, \ldots, p_{l}$ in $L$, one point $Q_{1}$ in $\mathbf{P}^{2}$ and a line $T_{1}$ through $Q_{1}$, we consider $T N_{\overrightarrow{1}}^{0}(1)$ (the closure of) the sublocus of $T N_{1}^{d, g}(1)$ of curves $C$ with $\sum_{i}(L \cdot C)_{p_{i}}=m$ and $T_{1}$ the common tangent line of two branches of $C$ at $Q_{1}$ which meet tangentially. Let $S$ be the blow-up of $\mathbf{P}^{2}$ at $p_{1}, p_{2}, \ldots, p_{l}$ as in the proof of (3.9). (Note that it was called $S_{m}$, but from now on, for the sake of notation we fix $S$ the blow-up of $\mathbf{P}^{2}$ at the fixed $l$ points $p_{i}$ in $L$.) Let $S_{1}$ be the blow-up of $S$ at $Q_{1}, E_{1}$ the exceptional curve of the blow-up $S_{1} \rightarrow S, Q_{1}^{\prime}$ the point on $E_{1}$ corresponding to $T_{1}$, and $S_{2}$ the blow-up of $S_{1}$ at $Q_{1}^{\prime}$. On $S_{2}$ the proper transform $\bar{C}_{2}$ of a general curve $C \in T N_{\overline{1}}^{0}(1)$ is equivalent to $\pi^{*} \bar{C}-2 \pi^{*} E_{1}-2 \pi^{*} E_{2}$ where $\bar{C}$ is the proper transform of $C$ of the blow-up $S \rightarrow \mathbf{P}^{2}$. Here $\pi$ represents blow-up maps from $S_{2}$ to $S_{1}, S$, or $\mathbf{P}^{2}$ according to context. Since $K_{S_{2}}=\pi^{*} K_{S}+\pi^{*} E_{1}+E_{2}$ (so, $-K_{S_{2}}$ is equivalent to an effective divisor $\pi^{*} H+\widetilde{T}_{1}+\widetilde{L}$ where $\widetilde{T}_{1}$ and $\widetilde{L}$ are the proper transforms of $T_{1}$ and $L$ respectively, and $H$ is a hyperplane divisor of $\left.\mathbf{P}^{2}\right),-K_{S_{2}} \cdot C_{2}=3 d-m-4$ which is always positive since $d$ is at least 4 for a plane curve of degree $d$ to have a tacnode. By Proposition (3.3) we have $\operatorname{dim} T N_{\overrightarrow{1}}^{0}(1)$ is at most $g+3 d-m-5$.

If $\kappa=2$, then $d$ is at least 5 . Adopting the previous setting we fix the second point $Q_{2}$ away from both $Q_{1}$ and $L$, and a line $T_{2}$ in general distinct from $T_{1}$ through $Q_{2}$. Now consider $T N_{2}^{0}(2)$ (the closure of) the sublocus of $T N_{2}^{d, g}(2)$ consisting of curves satisfying $\sum_{i}(C \cdot L)_{p_{i}}=m$ and each $T_{i}, i=1$ or 2 , is the common tangent line of two smooth branches of $C$ at $Q_{i}$. Blow up $S_{2}$ in the above at $Q_{2}$ twice as we did $S$ at $Q_{1}$, getting $S_{4}$. On $S_{4}$ we still have effective anticanonical divisor $-K_{4}$ having positive intersection number with $C_{4}$ the proper transform of a general member $C$. In fact by choosing $T$ the line through $Q_{1}$ and $Q_{2},-K_{4} \sim \widetilde{L}+2 \widetilde{T}+\widetilde{E}_{1}+\widetilde{E}_{3}$ and $-K_{4} \cdot C_{4}=3 d-m-8$, where $\sim$ representing the proper transform in $S_{4}$ of a corresponding divisor. Again Proposition (3.3) tells that

$$
\operatorname{dim} T N_{\overrightarrow{2}}^{0}(2) \leq g+3 d-m-9 \text { and } \operatorname{dim} T N_{2}^{0}(2) \leq g+3 d-m-7 .
$$

If $\kappa=3$ then $d \geq 5$ too. If we blow up all three tacnodes we will have trouble in making first the anticanonical divisor effective and second $3 d-m-12$ 
positive. Instead of fixing three tacnodes, therefore, we only fix two of them, say $Q_{1}$ and $Q_{2}$, together with lines $T_{i}$ through $Q_{i}$ as in the case $\kappa=2$. Then on $S_{4}$ we have a family of curves $C_{4}$ of genus $g$ and $C_{4} \cdot-K_{4}=3 d-m-8$, so of dimension at most $g+3 d-m-9$ with equality holding if and only if $C_{4}$ is a nodal curve. Since $C_{4}$ is not a nodal curve, the above family has dimension at most $g+3 d-m-10$, and then $g+3 d-m-4$ by removing the restrictions on $Q_{i}$. Thus $\operatorname{dim} T N_{3}^{d, g}(3) \leq g+3 d-m-10+l$. Q.E.D.

(3.11) Remark. If $\kappa \geq 2$ and one (or two) of $Q_{i}$ is (are) replaced by cusp(s) in (3.10) then on $S_{2}$ or on $S_{4}$, depending on the number of tacnodes, we do the same dimension counts as we did in the proof of (3.8) due to Proposition (3.6) to get the same upper bound of possible dimension of a corresponding locus.

This time we introduce the locus $U_{m, l}^{d, g}(\kappa)$ consisting of curves in $U^{d, g}$, a general member $C$ of which does not contain $L$, has one singular branch or two smooth branches meeting tangentially at each of the fixed $\kappa$ points $Q_{i}$, and for some collection of $l$ points $p_{1}, \ldots, p_{l}$ in $L$ satisfies $\sum_{i}(C \cdot L)_{p_{i}}=m$.

(3.12) Lemma. Let $W$ be a component of $U_{m, l}^{d, g}(\kappa)$ for $\kappa \leq 3$.

(1) (a) If $\kappa \geq 2$ and a general member $C$ of $W$ has two irreducible components which are tangent to each other at least at two points of $Q_{i}$, or $(\kappa=3) \quad C$ has three components each of which is tangent to the others (see (xiii) below), then

$$
\operatorname{dim} W \leq g+3 d-m-3 \kappa+l .
$$

(b) Otherwise; $\operatorname{dim} W \leq g+3 d-m-3 \kappa+l-1$.

(2) There is a corresponding parametric version of (1).

Proof. If a general member $C$ of a component $W$ of $U_{m, l}^{d, g}(\kappa)$ has a singular branch at each $Q_{i}$, see (3.9). If a general member $C$ is irreducible, see (3.10) and (3.11).

Now assume that a general curve $C$ is reducible. In this case we only consider the possible cases for $\kappa=3$. If $\kappa=1$ or 2 we will have much fewer cases than $\kappa=3$, which will eventually be included in what we will consider from now on. Note that it is enough to consider the components of $C$ through $Q_{i}$ because the remaining ones are in general of nodal curves with some contact with $L$ (cf. see the proof of (3.8) when $C$ is reducible and [H1, Lemma (2.5)]). Without further mention we assume that each component $C_{j}$ of $C$ has degree $d_{j}$ and genus $g_{j}$ with $\sum_{i}\left(C_{j} \cdot L\right)_{p_{i}}=m_{j}$ for some collection of $l$ points in $L$, where $\sum_{j} d_{j}=d, \sum_{j} g_{j}-n+1=g, n$ the number of components of $C$, and $\sum_{j} m_{j}=m$. Then $C$ is one of the following:

(a) $C$ has two components $C_{1}$ and $C_{2}$

(i) with $C_{1}$ having a cusp or a tacnode at each of $Q_{1}$ and $Q_{2}$, and $C_{2}$ a cusp or a tacnode at $Q_{3}$;

(ii) with $C_{1}$ having a cusp or a tacnode at $Q_{1}, C_{2}$ a cusp or a tacnode at $Q_{2}$, and $C_{1}$ and $C_{2}$ meeting tangentially at $Q_{3}$; 
(iii) with $C_{1}$ having a cusp or a tacnode at each of $Q_{1}$ and $Q_{2}$, and $C_{1}$ and $C_{2}$ meeting tangentially at $Q_{3}$;

(iv) with $C_{1}$ and $C_{2}$ meeting tangentially at $Q_{1}$ and $Q_{2}$, and $C_{1}$ having a cusp or a tacnode at $Q_{3}$;

(v) with $C_{1}$ and $C_{2}$ meeting tangentially at $Q_{1}, Q_{2}$ and $Q_{3}$.

(b) $C$ has three components $C_{1}, C_{2}$ and $C_{3}$

(vi) with each $C_{i}$ having a cusp or a tacnode at each $Q_{i}$;

(vii) with $C_{1}$ having a cusp or a tacnode at each of $Q_{1}$ and $Q_{2}$, and $C_{2}$ and $C_{3}$ meeting tangentially at $Q_{3}$;

(viii) with $C_{1}$ having a cusp or a tacnode at $Q_{1}$ and being tangent to $C_{3}$ at $Q_{3}$, and $C_{2}$ having a cusp or a tacnode at $Q_{2}$;

(ix) with $C_{1}$ having a cusp or a tacnode at $Q_{1}$ and meeting $C_{2}$ and $C_{3}$ tangentially at $Q_{2}$ and $Q_{3}$ respectively;

(x) with $C_{1}$ having a cusp or a tacnode at $Q_{1}$ and meeting $C_{2}$ tangentially at $Q_{2}$, and $C_{2}$ being tangent to $C_{3}$ at $Q_{3}$;

(xi) with $C_{1}$ and $C_{2}$ meeting tangentially at $Q_{1}$ and $Q_{2}$, and $C_{3}$ having a cusp or a tacnode at $Q_{3}$;

(xii) with $C_{1}$ meeting $C_{2}$ at $Q_{1}$ and $Q_{2}$ and $C_{3}$ at $Q_{3}$.

(xiii) with $C_{1}$ tangent to $C_{2}$ at $Q_{3}, C_{2}$ tangent to $C_{3}$ at $Q_{1}$ and $C_{3}$ tangent to $C_{1}$ at $Q_{2}$.

(c) $C$ has four components

(xiv) with $C_{i}, i=1$ or 2 , having a cusp or a tacnode at $Q_{i}$, and $C_{3}$ and $C_{4}$ meeting tangentially at $Q_{3}$;

(xv) with $C_{4}$ being tangent to $C_{1}$ and $C_{2}$ at $Q_{1}$ and $Q_{2}$ respectively, and $C_{3}$ having a cusp or a tacnode at $Q_{3}$;

(xvi) with $C_{1}$ having a cusp or a tacnode at $Q_{1}$ and meeting $C_{2}$ at $Q_{2}$ tangentially, and $C_{3}$ and $C_{4}$ meeting tangentially at $Q_{3}$;

(xvii) with $C_{1}$ and $C_{2}, C_{2}$ and $C_{3}, C_{3}$ and $C_{4}$ meeting tangentially at $Q_{1}, Q_{2}$ and $Q_{3}$ respectively;

(xviii) with $C_{4}$ being tangent to $C_{1}, C_{2}$ and $C_{3}$ at $Q_{1}, Q_{2}$ and $Q_{3}$ respectively;

(xvix) with $C_{1}$ and $C_{2}$ being tangent at $Q_{1}$ and $Q_{2}, C_{3}$ and $C_{4}$ at $Q_{3}$.

(d) $C$ has five components

(xx) with $C_{1}$ being tangent to $C_{2}$ at $Q_{1}, C_{3}$ to $C_{4}$ at $Q_{2}$, and $C_{5}$ having a cusp or a tacnode at $Q_{3}$;

(xxi) with $C_{4}$ being tangent to $C_{1}$ and $C_{2}$ at $Q_{1}$ and $Q_{2}$ respectively, and $C_{5}$ to $C_{3}$ at $Q_{3}$.

(e) $C$ has 6 components

(xxii) with $C_{1}$ being tangent to $C_{2}$ at $Q_{1}, C_{3}$ to $C_{4}$ at $Q_{2}$, and $C_{5}$ to $C_{6}$ at $Q_{3}$.

In cases (i) and (vi), all components meet transversely, so apply (3.9) or (3.10) to each $C_{i}$. 


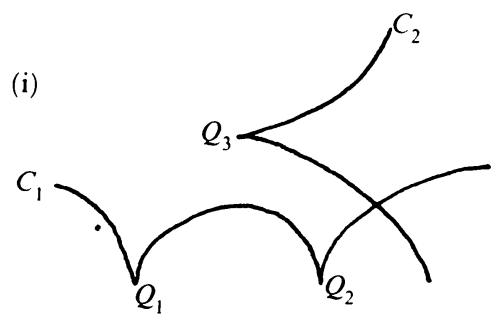

(iii)
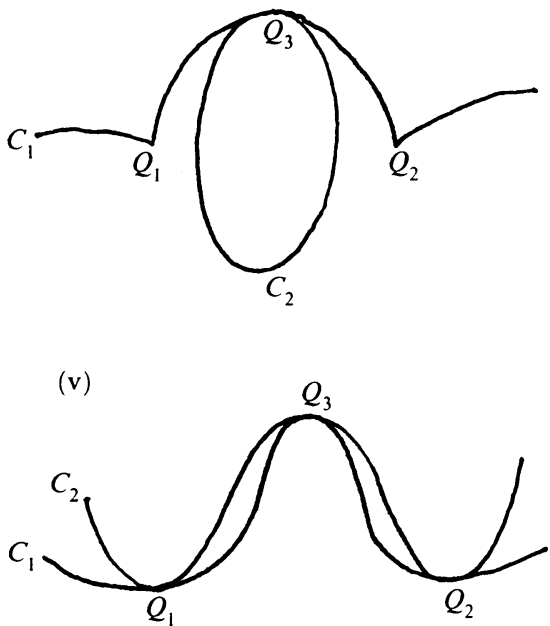

(vii)

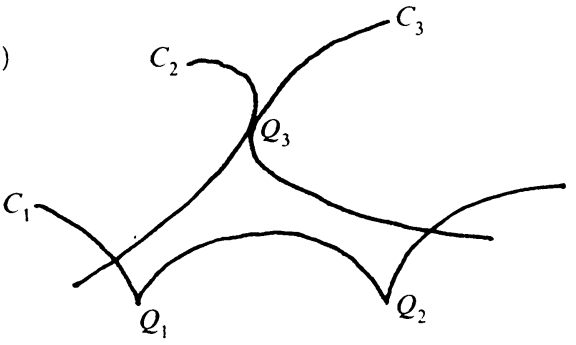

(ix)

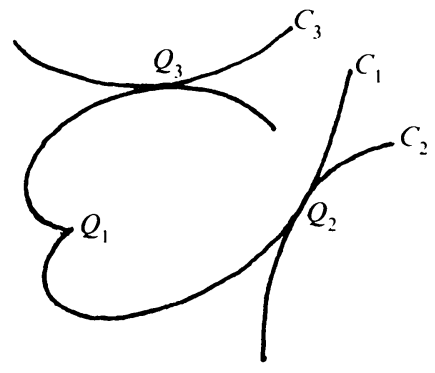

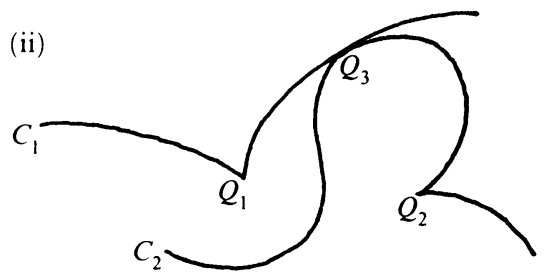

(iv)

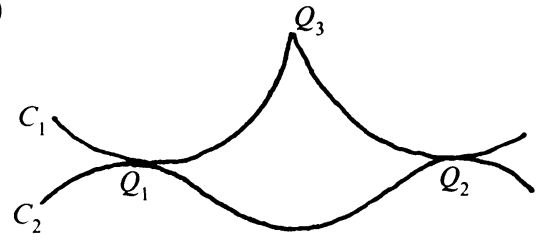

(vi)

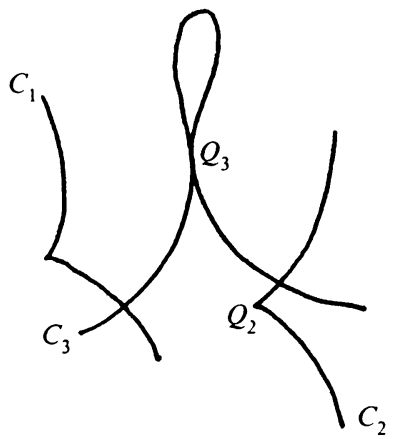

(viii)

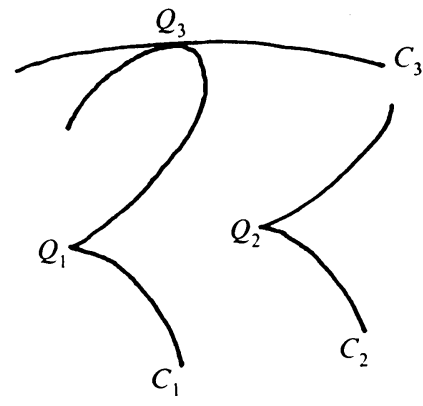

(x)

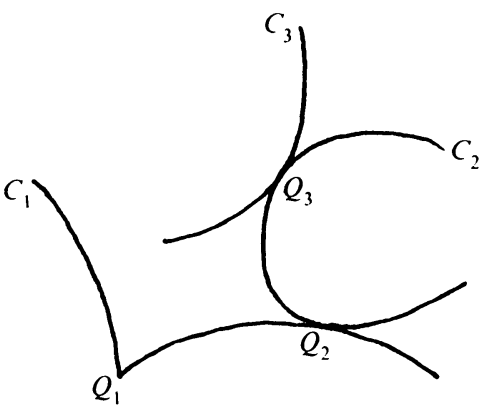



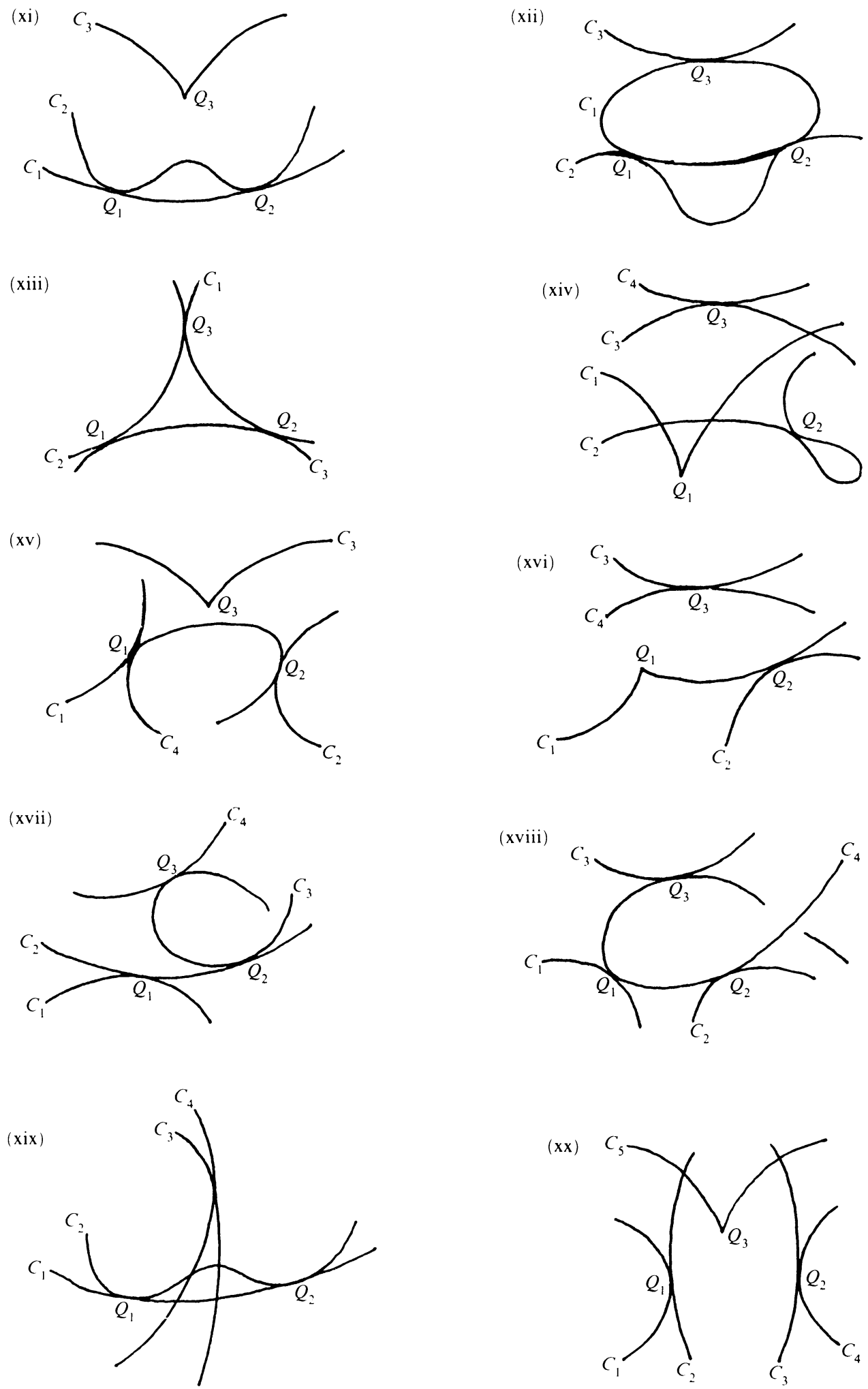
(xxi)

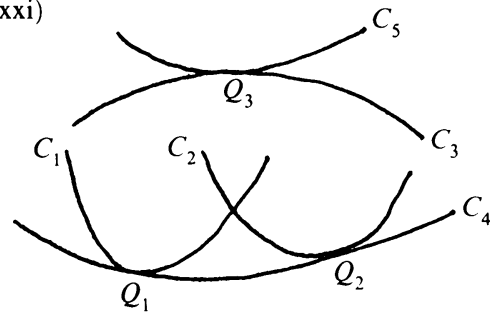

(xxii)

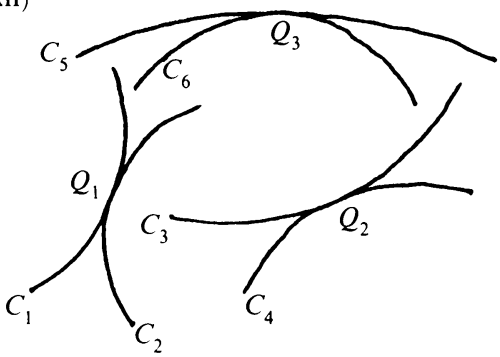

Proofs of (ii), (iii), (iv), (viii) and (ix) are similar; we blow up some of $Q_{i}$ without fixing others and use the existence of an automorphism. We only prove (ii) here. Choose a line $T_{3}$ through $Q_{3}$ and $l$ points $p_{i}$ in $L$. Blow up $\mathbf{P}^{2}$ at $p_{i}$ as in (3.9) and twice at $Q_{3}$ in the direction of $T_{3}$. We now apply (3.3) on this blown up space the anticanonical divisor of which is equivalent to an effective divisor and has an intersection number $3 d_{j}-m_{j}-2$ with the proper transform $\widetilde{C}_{j}$ of $C_{j}, j=1$ or 2 , to get that $C_{j}$ (without fixing $Q_{j}$ ) moves in a family of dimension at most $g_{j}+3 d_{j}-m_{j}-4$ since $\widetilde{C}_{j}$ is not a nodal curve. Therefore $C=C_{1} \cup C_{2}$ moves in a family of dimension at most $g+3 d-m+l-6$ by moving a line $T_{3}$ and $l$ points $p_{i}$ in $L$. Since there is an automorphism which maps $L$ to itself, fixes $Q_{3}$ and moves two points $\left(\neq Q_{3}\right.$ or $\left.\notin L\right)$ to another two points $\left(\neq Q_{3}\right.$ or $\left.\notin L\right)$, the curve $C$ in (ii) moves in a family of dimension at most $g+3 d-m+l-10$.

Proof of (vii). Consider $C_{1}$ as a curve in $V_{m_{1}, l_{1}, 2}^{d, g}, T N_{m_{1}, l_{1}, 2}^{d, g}(2)$ or in (3.11). Then $C_{1}$ moves in a family of dimension at most $g_{1}+3 d_{1}-m_{1}-7+l_{1}$. With each $C_{1}, l_{1}(\leq l)$ points $p_{i} \in L$ are chosen. If $l_{1}<l$, choose extra $l-l_{1}$ points in $L$. We may assume that $d_{2} \leq d_{3}$ (then $d_{2}$ is possibly 1 and $d_{2} \geq 2$ ). Then $C_{2}$ as a curve simply through $Q_{3}$ with some contact with $L$ moves in a family of dimension at most $g_{2}+3 d_{2}-m_{2}-2$ and $C_{3}$ with the tangent line of $C_{2}$ at $Q_{3}$ in a family of dimension at most $g_{3}+3 d_{3}-m_{3}-3$. Therefore $C$ moves in a family of dimension at most $(g+2)+3 d-m-12+l_{1}+\left(l-l_{1}\right)$.

Proofs of (x), (xv), (xvi), (xvii), and (xviii) are similar too. We only give the proof of (xvi). Consider $C_{1}$ as a curve that has a cusp at $Q_{1}$ and passes through $Q_{2}$. Then applying (3.3) on the blow-up of $S$ at $Q_{1}$ as in (3.8.1) and once at $Q_{2}$ we get that $C_{1}$ moves in a family of dimension at most $g_{1}+3 d_{1}-m_{1}-5$; with the tangent line of each $C_{1}$ at $Q_{2}$ we see that $C_{2}$ moves in a family of dimension at most $g_{2}+3 d_{2}-m_{2}-3$ (again if $C_{2}$ is a line then it is the tangent line of each $C_{1}$ in general missing preassigned $l$ points in $L$ ); similarly $C_{3}$ moves in a family of dimension at most $g_{3}+3 d_{3}-m_{3}-2$ and $C_{4}$ in a family of dimension at most $g_{4}+3 d_{4}-m_{4}-3$.

Proofs of (xiv), (xx), (xxi) and (xxii): with preassigned $l$ points in $L$ we take care of each $C_{j}$ in appropriate order. We here prove (xiv). By (3.9) or (3.10), $C_{j},\left(j=1\right.$ or 2 , moves in a family of dimension at most $g_{j}+3 d_{j}-m_{j}-4$. 
For $C_{3}$ and $C_{4}$ we do exactly the same thing as we have done for $C_{2}$ and $C_{3}$ in case (vii).

Proof of (xiii). Assume $d_{1} \leq d_{2} \leq d_{3}$. If $C_{1}$ is a line through $Q_{2}$ and $Q_{3}$, then $d_{2}$ and $d_{3}$ are at least 2. Blowing up $S$ twice at $Q_{2}$ (at $Q_{3}$ resp.) and once at $Q_{1}, C_{3}\left(C_{2}\right.$ resp.) moves in a family of dimension at most $g_{3}+3 d_{3}-$ $m_{3}-4\left(g_{2}+3 d_{2}-m_{2}-4\right.$ resp.). Since we may assume that $C_{1}$ misses preassigned $p_{i} C$ moves in a family of dimension at most $(g+2)+3(d-1)-m-8$. (In fact we can get dimension bound as $g+3 d-m-10$ unless both $C_{2}$ and $C_{3}$ are conics.)

In general we may assume that $2 \leq d_{1} \leq d_{2} \leq d_{3}$. Then $C_{1}$ as a curve through $Q_{2}$ and $Q_{3}$ moves in a family of dimension at most $g_{1}+3 d_{1}-m_{1}-3$. Now choose the tangent lines $T_{j}$ of $C_{1}$ at $Q_{j}, j=2,3$. Then $C_{2}\left(C_{3}\right.$ resp.) moves in a family of dimension at most $g_{2}+3 d_{2}-m_{2}-4\left(g_{3}+3 d_{2}-m_{3}-4\right.$ resp.): blow up $S$ twice at $Q_{3}$ ( $Q_{2}$ resp.) in the direction of $T_{3}\left(T_{2}\right.$ resp.) and once at $Q_{1}$. Again we can have $g+3 d-m-10$ unless all three are conics.

Proofs of the remaining cases (v), (xi), (xii) and (xix). These are the cases in which two components of $C$ meet tangentially at least at two points. The trouble only happens when both curves are conics ((xi), (xii), (xiii)) or when one is a conic and the other is a cubic ( $\mathrm{v})$; otherwise we can prove similarly as case (xvi). We here give the proof of (xi), but the proofs of the others are very similar.

Proof of (xi). Choose $l$ points $p_{i}$ in $L$ and let $S$ be the blow-up of $\mathbf{P}^{2}$ at $p_{i}$ as in (3.9) or (3.10). If $C_{1}$ is a line then $C_{2}$ has degree at least 4. So $C_{2}$ moves in a family of curves of dimension at most $g_{2}+3 d_{2}-m_{2}-5$ : apply (3.3) to the blow-up of $S$ twice both at $Q_{1}$ and at $Q_{2}$ in the direction of the line through $Q_{1}$ and $Q_{2}$. Since $C_{3}$ has one cusp or one tacnode, by (3.9) or (3.10) $C_{3}$ moves in a family of curves of dimension at most $g_{3}+3 d_{3}-m_{3}-4$, and thus $C$ in a family of dimension at most $g+3 d-m-10$ with $l$ points in $L$ fixed.

In general assume that $2 \leq d_{1} \leq d_{2}$. As a curve through $Q_{1}$ and $Q_{2}$ with $\sum_{i}\left(C_{1} \cdot L\right)_{p_{i}}=m_{i}, C_{1}$ moves in a family of dimension at most $g_{1}+3 d_{1}-m_{1}-3$ : apply (3.3) to the blow-up of $S$ at $Q_{1}$ and $Q_{2}$. Now fix only one tangent line $T_{1}$ of $C_{1}$ at $Q_{1}$. Then $C_{2}$, as a curve tangent to $T_{1}$ and through $Q_{2}$, moves in a family of dimension at most $g_{2}+3 d_{2}-m_{2}-4$ (in fact, $g_{2}+3 d_{2}-m_{2}-5$ unless $d_{2}=m_{2}=2$ ), therefore $C$ in a family of dimension at most $g+3 d-m-9$ with $l$ points in $L$ fixed. Q.E.D.

The dimension of the locus we will need finally in the next section is the following: let $T_{m, l, k}^{d, g}$ be the locus of reduced plane curves $C$ in $U^{d, g}$ which have an $n$-tuple point at $Q_{1}$ and singular points at the remaining $Q_{i}$, do not contain the line $L$, and satisfy $\sum_{i}(C \cdot L)_{p_{l}}=m$ for some collection of $l$ points $p_{1}, \ldots, p_{l}$ in $L$. Then

(3.13) Lemma. (1) (a) $\operatorname{dim} T_{m, l, \kappa}^{d, g} \leq g+3 d-m-2 \kappa-n+l+1$. 
(b) Let $W$ be a component of $T_{m, l, k}^{d, g}$ whose members $C$ have a singular branch at each $Q_{i}$ for $i \geq 2$, then

$$
\operatorname{dim} W \leq g+3 d-m-n-3(\kappa-1)+l-1 .
$$

In particular, if $n=3, \operatorname{dim} W \leq g+3 d-m-3 \kappa+l-1$.

(2) There is a corresponding parametric version.

Proof. We may assume that $C$ does not contain lines through two of $Q_{i}$; in this case the lines are fixed, so we may assume that these lines would miss the preassigned $p_{i}$ or that some $p_{i}$ are fixed by these lines among $l$ points in $L$. Choose $l$ points in $L$. Let $S$ be the blow-up of $\mathbf{P}^{2}$ at $p_{i}$ as in (3.9). Let $S_{3}$ be the blow-up of $S$ at $Q_{1}, Q_{2}$ and $Q_{3}$. Then the proper transform of any component of $C$ has positive intersection number with the anticanonical divisor of $S_{3}$. Applying (3.3), we get $\operatorname{dim} T_{m, l, \kappa}^{d, g} \leq g+3 d-m-2 \kappa-n+1$ since $-K_{3} \cdot \widetilde{C}=3 d-m-n-2(\kappa-1), \widetilde{C}$ the proper transform of $C$ in $S_{3}$.

To show (b), we blow up $S$ only at $Q_{1}$ without fixing the remaining $Q_{i}$. Let $S_{1}$ be the blow-up of $S$ at $Q_{1}$. Then it can be easily checked that any component of the proper transform of $C$ satisfies the condition of (3.6). Applying (3.6) to each component and adding each possible dimension, we conclude that $C$, without fixing $Q_{i}$ for $i \geq 2$, moves in a family of dimension at most $g+3 d-m-n-(\kappa-1)-1$ since $-K_{S_{1}} \cdot \widetilde{C}=3 d-m-n$ and $\bar{m}(\widetilde{C})=\kappa-1$, $\widetilde{C}$ the proper transform of $C$ in $S_{1}$. Moving first $l$ points in $L$ and using an automorphism of $\mathbf{P}^{2}$, we have $\operatorname{dim} W \leq g+3 d-m-n-3(\kappa-1)+l-1$.

\section{Proof of Theorem (1.2)}

Due to Theorems (2.1), (2.2) and Remark (2.3), Theorem (1.2) will follow once we show that any component $W$ of $V(d, \delta, \kappa)$ contains in its closure a component of $V\left(d, \frac{1}{2}(d-1)(d-2)-\kappa, \kappa\right)$.

For this purpose we first show the following proposition asserting the existence of certain degenerations in the families $V_{m, \kappa}(d, \delta, \kappa)$ when $\kappa \leq 3$, where $V_{m, \kappa}(d, \delta, \kappa)=V(d, \delta, \kappa) \cap V_{m, \kappa}^{d, g}$, i.e., an open dense set of $V_{m, \kappa}^{d, g}$.

(4.1) Proposition. Let $W$ be a component of $V_{m, \kappa}(d, \delta, \kappa)$. Then $W$ contains in its closure either

(1) a component of $V_{m+1, \kappa}(d, \delta, \kappa)$; or

(2) a component of $U_{m, k}^{d, g-1}$ whose general member is a curve $C$ that either

(a) is smooth at its point of intersection multiplicity $m$ with $L$, and has at most two irreducible components; or

(b) (if $m \geq 2$ ) has exactly two branches at its unique point $p$ of intersection multiplicity $m$ with $L$, with each of its (at most two) irreducible components containing $p$. 
Proof. Let $X$ be the intersection of the closure of $W$ with $g+3 d-3 \kappa-m-1$ general hypersurfaces in the space $\mathbf{P}^{N}$ of curves of degree $d$. Applying the semistable reduction theorem we may make a series of finite base changes $B \stackrel{\alpha}{\longrightarrow}$ $X$ and blow-ups and blow-downs to arrive at a 1-parameter family $\pi: \Xi \rightarrow B$ of nodal curves of arithmetic genus $g$, with smooth total space $\Xi$, a map $\eta: \Xi \rightarrow \mathbf{P}^{2}$ mapping the fibers $X_{b}=\pi^{-1}(b)$ of $\pi$ to the corresponding plane curves $\alpha(b) \in X$, and $\Xi$ minimal with respect to these properties; i.e., there are no rational components of fibers of $\pi$ meeting the rest of the fibers in only one point and on which $\eta$ is constant. If $m>1$, for general $b \in B$, then $\eta^{*}(L) \mid X_{b}$ will have single point $p_{b}$ of multiplicity $m$; by the assumption that $\Xi$ is smooth, these points will extend to a section $\Gamma$ of $\pi$. If $m=1$, we can (after possibly further base changes, blowing-ups and blowing-downs) similarly arrive at a section $\Gamma$ of $\pi$ meeting the general fiber $X_{b}$ in a point of $\eta^{-1}(L)$. There are also $\kappa$ sections $\Gamma_{i}$ of $\pi$ for $1 \leq i \leq \kappa$ : for general $b \in B, X_{b}$ will have single point $q_{b_{i}}$ which maps to $Q_{i}$ and again these points will extend to a section $\gamma_{i}$ of $\pi$ since $\Xi$ is smooth.

Case (i). All the fibers of $\pi$ are smooth.

In this case we claim that $W$ must contain in its closure a component of $V_{m+1, \kappa}(d, \delta, \kappa)$ of genus $g$. To see this, write the divisor $\eta^{*} L$ as $\eta^{*} L=m \Gamma+$ $\Gamma^{\prime}$. Note that we cannot have $\Gamma^{\prime}=0$, since $\eta^{*} L$ has positive self intersection and $\Gamma^{2}$ nonpositive (cf. [H4]). On the other hand $\eta^{*} L$ must be connected [Ha, Chapter 3, Exercise 11.3], so $\Gamma \cap \Gamma^{\prime} \neq \varnothing$. Now, for any $b_{0} \in \pi\left(\Gamma \cap \Gamma^{\prime}\right)$, we have mult $_{p_{b_{0}}}\left(\left.\eta^{*} L\right|_{X_{b_{0}}}\right) \geq m+1$. Thus the map $\eta \mid X_{b_{0}}$ will have contact of order at least $m+1$ with $L$ at $p_{b_{0}}$. (See [H1] for the above.)

Also each $\Gamma_{i}$ meets $X_{b_{0}}$ at one point $q_{i}$ because all the fibers are smooth. Since $\eta$ is regular, $C_{0}=\eta\left(X_{b_{0}}\right)$ has at least one branch with multiplicity at least 2 at $Q_{i}=\eta\left(q_{i}\right)$. So, $\bar{m}_{Q_{i}}\left(C_{0}\right) \geq 1$. Therefore $C_{0}$ moves in a family $V_{m+1, \kappa}^{d, g}$ of dimension $g+3 d-3 \kappa-(m+1)$ and we conclude from Lemma (3.8) that $C_{0}$ is a general member of a component of $V_{m+1, \kappa}^{d, g}$ which is a nice curve with cusps at $Q_{i}$ and has a contact of order $m+1$ with $L$ at a smooth point of $C_{0}$.

Case (ii). Some fiber $X_{0}$ of $\pi$ is singular.

Let $\mathscr{L}$ be the pullback line bundle $\eta^{*} \mathscr{O}_{\mathbf{p}^{2}}(1)$. Let $p_{0} \in X_{0}$ be the point of intersection $X_{0} \cap \Gamma$ (Note that $p_{0}$ is a smooth point since $\Xi$ is smooth.) Let $Y_{0}^{\prime} \subset X_{0}$ be the connected component of $\eta^{-1}(L) \cap X_{0}$ containing $p_{0}$; this will either be $p_{0}$ itself or a union of irreducible components of $X_{0}$. Let $Y_{0}$ be the closure of the complement of $Y_{0}^{\prime}$ in $X_{0}$ and write $Y_{0}^{\prime} \cap Y_{0}=\left\{p_{1}, \ldots, p_{k}\right\}$ (so that, in particular, if $Y_{0}^{\prime}=\left\{p_{0}\right\}$, then $k=1$ and $p_{1}=p_{0}$ ). Let $a_{i}$ be the multiplicity mult $p_{i}\left(\left.\eta^{-1}(L)\right|_{Y_{0}}\right)$ with which $p_{i}$ appears in the divisor $\eta^{-1}(L)$ 
restricted to $Y_{0}$ and let $n=\sum_{i=1}^{k} a_{i}$. Write

$$
\operatorname{deg}\left(\left.\mathscr{L}\right|_{Y_{0}^{\prime}}\right)=\alpha, \quad \operatorname{deg}\left(\left.\mathscr{L}\right|_{Y_{0}}\right)=d-\alpha
$$

and let $\beta$ be the sum of the degrees of $\mathscr{L}$ on the components of $Y_{0}$ in $\eta^{-1}(L)$. Then from [H1], we know $g\left(y_{0}\right) \leq p_{a}\left(y_{0}\right) \leq g-k+1$ with equality holding only if $Y_{0}$ is smooth and $Y_{0}^{\prime}$ is of arithmetic genus zero. Secondly $n \geq \eta^{-1}(L) \cdot Y_{0} \geq$ $m-\alpha$.

In addition to the above we consider $\eta^{-1}\left(Q_{i}\right)$ or $\Gamma_{i}$. Since $\left(\Gamma_{i} \cdot X_{0}\right)=1$, each $\Gamma_{i}$ meets $Y_{0}$ at a smooth point $q_{i}$ away from components of $\eta^{-1}(L)$. Let $Y_{i}^{\prime}$ be the connected component of $\eta^{-1}\left(Q_{i}\right) \cap X_{0}$ containing $q_{i}$.

Case (iia). Assume $Y_{i}^{\prime}=\left\{q_{i}\right\}=\Gamma_{i} \cap X_{0}$ for all $i$. Then as in the case (i), $\bar{m}_{Q_{i}}\left(C_{0}\right) \geq 1$.

Let $Y$ be the disjoint union of the normalizations of the components of $Y_{0}$ not contained in $\eta^{-1}(L)$ on which $\eta$ is nonconstant. Write $\eta: Y \rightarrow \mathbf{P}^{2}$ for the composite $Y \rightarrow Y_{0} \rightarrow \mathbf{P}^{2}$. The image of $\eta$ is of degree $d-\alpha-\beta$ and has $k$ points with a total order of contact with $L$ at least $m-\alpha$ and $\bar{m}_{Q_{i}}(\eta(Y)) \geq 1$ since $Q_{i} \notin L$. Thus, by Lemma (3.9), map $\left.\eta\right|_{Y}$ moves in a family of dimension at most $3(d-\alpha-\beta)+(g-k+1)-3 \kappa-(m-\alpha)+k-1$ which we expect is not less than $3 d+g-3 \kappa-m-1$. Thus $-2 \alpha-3 \beta \geq-1$, which implies that $\alpha=\beta=0$.

Having $\alpha=0$, all of $Y_{0}^{\prime}$ must map to the point $\eta\left(p_{0}\right)=p$ and, from the minimality of $\Xi, Y_{0}^{\prime}$ is not of arithmetic genus zero when $k=1$. Thus $\eta(Y)$ moves in a family $U_{m, k}^{d, g_{1}}$ of dimension at most $3 d+g_{1}-3 k-m$ where $g_{1}=\operatorname{genus}\left(Y_{0}\right) \leq \min (g-1, g-k+1)$. By dimension count of a family of $\eta(Y)$ again by Lemma (3.8) we have

$$
3 d+g_{1}-3 \kappa-m \geq 3 d+g-3 \kappa-m-1 .
$$

Therefore $g_{1}=g-1$ and $k \leq 2$. Having an equality in $(*), \eta(Y)$ is a general member of a component of $U_{m, \kappa}^{d, g-1}$ having intersection number $m$ with $L$ at a point $p$. If $k=1, p$ is a smooth point of $\eta(Y)$ and $\eta(Y)$ is simply a curve of degree $d$ with $\delta+1$ nodes and $\kappa$ cusps at $Q_{i}$ as its only singularities; if $k=2, \eta(Y)$ has a double point at $p$ consisting of two smooth branches with contact of order $a, \kappa$ cusps at $Q_{i}, \delta+1-a$ nodes elsewhere, and no other singularities. In any case $\eta(Y)$ has at most two components otherwise $X_{0}$ would drop its genus at least by 2 . In particular $Y_{0}$ is smooth, so if $Y_{0}$ has two components then they are disjoint but meet $Y_{0}^{\prime}$ since $X_{0}$ is connected. Both components of $\eta(Y)$ thus go through $p$.

Case (iib). $X_{0}$ is singular and $Y_{i}^{\prime} \supsetneqq\left\{q_{i}\right\}$ for some $i$.

Say $i=1$. As we did in the analysis of $\eta^{-1}(L)$, let $Y_{1}^{\prime}$ be the connected component of $\eta^{-1}\left(Q_{1}\right) \cap Y_{0}$ containing $q_{1}$; since we are assuming $Y_{1}^{\prime} \supsetneqq\left\{q_{1}\right\}$, 
this is a union of irreducible components of $X_{0}$. Let $Y_{1}$ be the closure of the complement of $Y_{1}^{\prime}$ in $Y_{0}$. Say $Y_{1}^{\prime} \cap Y_{1}=\left\{q_{1}^{1}, \ldots, q_{1}^{n_{1}}\right\}$. Then

$$
g\left(Y_{1}\right) \leq p_{a}\left(Y_{1}\right)=p_{a}\left(Y_{0}\right)-p_{a}\left(Y_{1}^{\prime}\right)-n_{1}+1 \leq p_{a}\left(Y_{0}\right)-n_{1}+1
$$

equality only if $Y_{1}$ is smooth and $Y_{1}^{\prime}$ is rational. Note that $Y_{1}^{\prime}$ is not rational when $n_{1}=1$ due to the minimality of $\Xi$. So,

$$
g\left(Y_{1}\right) \leq \min \left\{p_{a}\left(Y_{0}\right)-1, p_{a}\left(Y_{0}\right)-n_{1}+1\right\} \leq g-k-n_{1}+2 .
$$

(1) Assume $n_{1}=1$. Then $Y_{1}^{\prime}$ is not rational because of the minimality of $\Xi$. The picture of $X_{0}$ in this case looks like the following: We therefore see that there is a branch of $C_{0}=\eta\left(X_{0}\right)$ through $Q_{1}$ on which $\bar{m}_{Q_{1}} \geq 1$ : look at a small neighborhood $Z$ of $q_{1}^{1}$, then mult $_{\eta\left(q_{1}^{1}\right)}(\eta(Z)) \geq 2$, and therefore $\bar{m}_{Q_{1}}^{\prime}(\eta(Z)) \geq 1$.

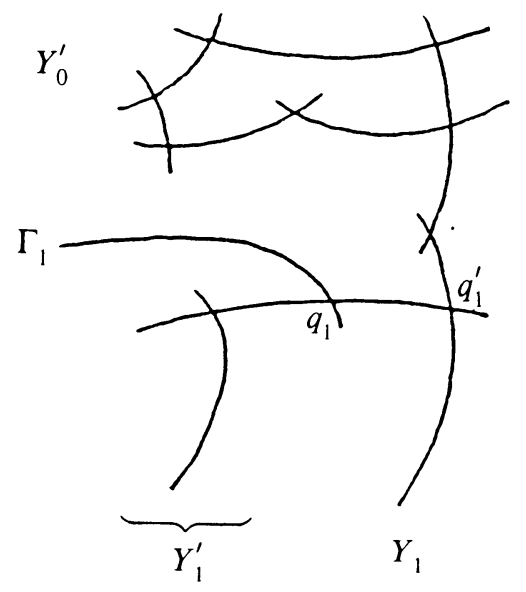

(2) Assume $n_{1}=2$. Then $X_{0}\left(\right.$ near $\left.\Gamma_{1}\right)$ looks like either one of the following two pictures.
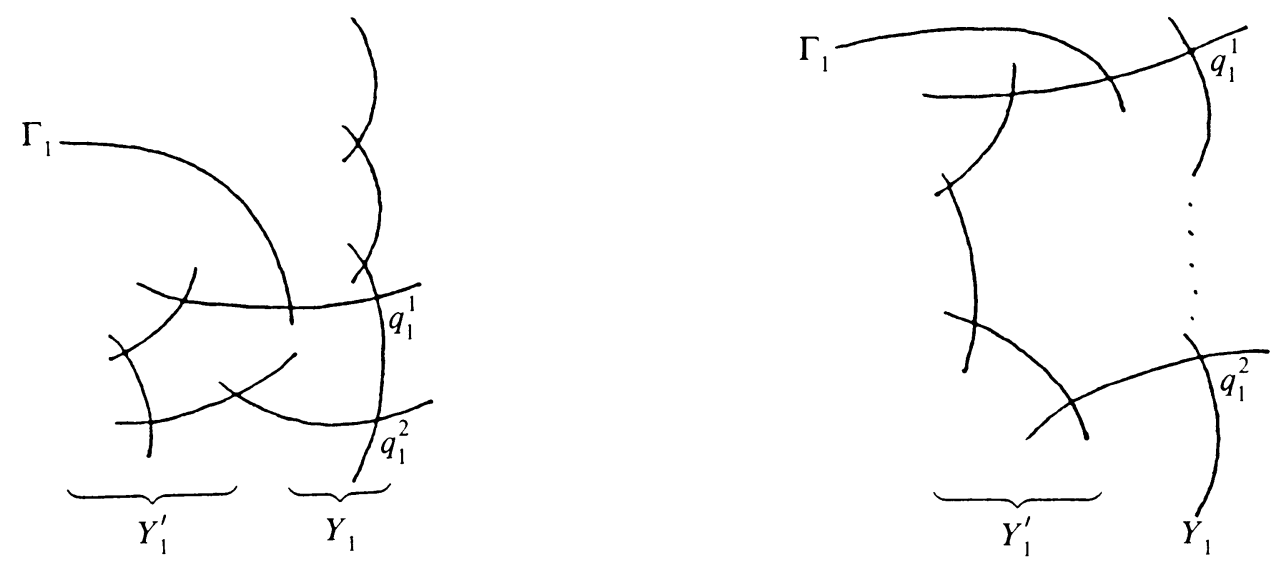
Now take a small neighborhood $N_{i}$ of $q_{1}^{i}$ in $Y_{1}$ on which $q_{1}^{i}$ is the only point of $\eta^{-1}\left(Q_{1}\right)$ respectively. Assume $N_{1} \cap N_{2}=\varnothing$; if the image of any of $N_{i}$ is not smooth at $Q_{1}$, then $\bar{m}_{Q_{1}}\left(\eta\left(N_{i}\right)\right) \geq 1$; if the images of both $N_{i}$ are smooth, then they meet at $Q_{1}$ with contact at least two since a cusp cannot be degenerated to a node.

(3) Let $n_{1} \geq 3$. We consider $N_{i}$ as before at $q_{1}^{i}$ and assume $N_{i} \cap N_{j}=\varnothing$ for $i \neq j$. If one of them has a nonsmooth image at $Q_{1}$, then $\bar{m}_{Q_{i}}\left(C_{0}\right) \geq 1$. If all the images of $N_{i}$ are smooth at $Q_{1}$, then either two of them meet with contact at least two at $Q_{1}$ or all of them meet transversely. So the only new case we see here is that the map $\left.\eta\right|_{X_{0}}$ has a triple point (in general an $n_{1}$-tuple point) at $Q_{1}$.

If $Y_{1}$ is not smooth and $Y_{i}^{\prime} \supsetneqq\left\{q_{i}\right\}$ for some $i \geq 2$ in any possibility of case (iib), do the same analysis we have done here; starting with $Y_{1}$, getting $Y_{2}^{\prime}, Y_{2}$ and $n_{2}=\#\left\{Y_{2}^{\prime} \cap Y_{2}\right\}$, and then in $Y_{2}$ (if necessary) $Y_{3}^{\prime}, Y_{3}$ and $n_{3}$. Then

$$
(* * *) \quad g\left(Y_{2}\right) \leq p_{a}\left(Y_{2}\right) \leq \min \left\{p_{a}\left(Y_{1}\right)-1, p_{a}\left(Y_{1}\right)-n_{2}+1\right) \leq g-k-n_{1}-n_{2}+3 \text {. }
$$

Except for the case that at least two of $Q_{i}$ lie in (2) or (3) of case (iib) and $\eta\left(X_{0}\right)$ admits no singular branches at $Q_{2}$ or $Q_{3}$, let $Y$ be the disjoint union of the normalizations of the components of $Y_{1}$ not contained in $\eta^{-1}(L)$ or $\eta^{-1}\left(Q_{1}\right)$ on which $\eta$ is not constant. Write $\eta: Y \rightarrow \mathbf{P}^{2}$ for the composite $Y \rightarrow Y_{1} \rightarrow \mathbf{P}^{2}$. Then the map $\left.\eta\right|_{Y}$ lies in one component of the closure of the locus described in (3.12)(b) if $n_{1} \leq 2$ or in (3.13)(b) if $n_{1} \geq 3$ with degree $d-\alpha-\beta$ and genus at most $g-k-n_{1}+2$, therefore moves in a family of dimension at most

$$
\left(g-k-n_{1}+2\right)+3(d-\alpha-\beta)-3 \kappa-(m-\alpha)+k-1,
$$

which we expect is at least $g+3 d-3 \kappa-m-1$. Thus $-2 \alpha-3 \beta \geq n_{1}-2 \geq-1$ since $n_{1} \geq 1$, which implies that $\alpha=\beta=0$. Having $\alpha=0, k$ points of $Y_{0}^{\prime} \cap Y_{0}$ are mapped to one point $\eta\left(p_{0}\right)$ and, due to the minimality of $\Xi, Y_{0}^{\prime}$ is not arithmetic genus zero when $k=1$, so $g\left(Y_{0}\right) \leq \min (g-1, g-k+1)$ and $g\left(Y_{1}\right) \leq \min \left(g-2, g-k-n_{1}+2\right)$ (see $\left.(* *)\right)$. Letting $g_{1}$ the genus of $Y_{1}$ and counting dimension again we get $g_{1} \geq g-1$, which is impossible.

In the case we have to consider $Y_{2}$, let $Y$ be the disjoint union of the normalization of the components of $Y_{2}$ not contained in $\eta^{-1}(L)$ or $\eta^{-1}\left(Q_{i}\right)$ for $i=1,2$, on which $\eta$ is not constant. Write $\eta: Y \rightarrow \mathbf{P}^{2}$ for the composite $Y \rightarrow Y_{2} \rightarrow \mathbf{P}^{2}$. We may assume $n_{1} \geq n_{2} \geq 2$. If $n_{1}=n_{2}=2$, we can say that $\eta(Y)$ has tacnodes at $Q_{1}$ and $Q_{2}$, and has a singular point at $Q_{3}$ other than a node from our previous analysis. By (3.12)(a), it moves in a family of curves of dimension at most

$$
\begin{gathered}
\left(g-k-n_{1}-n_{2}+3\right)+3(d-\alpha-\beta)-3 \kappa-(m-\alpha)+k \\
\geq g+3 d-3 \kappa-m-1 .
\end{gathered}
$$


So

$$
-2 \alpha-3 \beta \geq n_{1}+n_{2}-4=0 .
$$

Thus $\alpha=\beta=0$ and $k$ points of $Y_{0}^{\prime} \cap Y_{0}$ are mapped to one point $\eta\left(p_{0}\right)$. As before, $g\left(Y_{0}\right) \leq \min (g-1, g-k+1)$ and $g\left(Y_{2}\right) \leq \min \left(g-3, g-k-n_{1}-n_{2}+3\right)$ (see $(* * *)$ ). Letting $g_{2}=g\left(Y_{2}\right)$ and counting dimension again, we get $g_{2} \geq$ $g-2$, which is a contradiction. If $n_{1} \geq 3$ and $n_{2} \geq 2$, we may now assume that the map $\left.\eta\right|_{Y}$ admits a triple point at $Q_{1}$ and a tacnode at $Q_{2}$ due to the dimension counts in the last section. Then the map $\left.\eta\right|_{Y}$ moves in a family of dimension at most

$$
\begin{gathered}
\left(g-k-n_{1}-n_{2}+3\right)+3(d-\alpha-\beta)-(m-\alpha)-2 \kappa-2+k \\
\geq g+3 d-m-3 \kappa-1 .
\end{gathered}
$$

So $-2 \alpha-3 \beta \geq n_{1}+n_{2}-\kappa-2 \geq 0$ (in fact, it is only worth considering when $\kappa=3)$. Thus $\alpha=\beta=0$ as in the previous cases. But if $n_{1} \geq 3$ and $n_{2} \geq 2$, $g_{2}=g\left(Y_{2}\right) \leq \min \left(g-1-n_{1}-n_{2}+2, g-k-n_{1}-n_{2}+3\right) \leq g-4$. New dimension count shows that $g_{2} \geq g-3$.

The only possibilities in case (ii) therefore are the ones that occur in (iia).

(4.2) To complete the proof, we follow the same argument as Harris used in $[\mathrm{H} 1, \S 4]$ for $V^{d, g}$. Let $X \subset \mathbf{P}^{N}$ be any closed family of plane curves whose general member is reduced and irreducible of geometric genus $g$ having cusps at the fixed points. We have then a rational map $\varphi=\varphi_{X}: X \rightarrow \overline{\mathfrak{M}}_{g}$ where $\overline{\mathfrak{M}}_{g}$ is the moduli space of stable curves of genus $g$. We denote $\Phi=\Phi_{X} \subset$ $X \times \overline{\mathfrak{M}}_{g}$ the graph of this map and by $\varphi_{1}, \varphi_{2}$ the projections of $\Phi$ to $X$ and $\overline{\mathfrak{M}}_{g}$. Let $\Delta_{0} \subset \overline{\mathfrak{M}}_{g}$ be the closure of the locus of irreducible singular curves. We say that the family $X$ degenerates to $\Delta_{0}$ if $\varphi(X)$ meets $\Delta_{0}$, and that $X$ degenerates to $\Delta_{0}$ in codimension one in cusp preserving manner if the inverse image $\varphi^{-1}\left(\Delta_{0}\right)=\varphi_{1}\left(\varphi_{2}^{-1}\left(\Delta_{0}\right)\right)$ in $X$ of $\Delta_{0}$ has codimension one in $X$ and a general element of some component of $\varphi^{-1}\left(\Delta_{0}\right)$ has cusps at the limits of cusps of the general member of $X$.

We observe that since a multiple of the divisor $\Delta_{0}$ in $\overline{\mathfrak{M}}_{g}$ is Cartier, $\varphi^{-1}\left(\Delta_{0}\right)$ can fail to be of codimension one in $X$ only if it is empty or if the general fiber of $\varphi^{-1}\left(\Delta_{0}\right)$ over its image in $X$ is positive dimensional. It follows that $X$ degenerates to $\Delta_{0}$ in codimension one in cusp preservng manner whenever there is an isolated point $(C, D)$ of $\varphi_{2}^{-1}\left(\Delta_{0}\right) \cap \varphi_{1}^{-1}(C)$ and $C$ has cusps at the limits of the points where the general member of $X$ has cusps.

One circumstance in which this will be the case is the following: After making a finite base change $\alpha: B \rightarrow \Phi$ there will exist a family $\pi: \Xi \rightarrow B$ of stable curves and a rational map $\eta: \Xi \rightarrow \mathbf{P}^{2}$ carrying a general fiber $X_{b}=\pi^{-1}(b)$ of $\pi$ to the corresponding plane curve. Suppose now that for some point $\left(C_{0}, D_{0}\right) \in \Phi$ the map $\eta_{\mid D_{0}}: D_{0} \rightarrow C_{0}$ is defined and birational on every component of $D_{0}$ except possibly rational curves containing three or fewer branches 
of nodes of $D_{0}$, having cusps at the limits of cusps of the general member of $X$-for example, $D_{0} \rightarrow C_{0}$ is just a partial normalization with cusps of $C_{0}$ desingularized in $D_{0}$. Then clearly we cannot vary the isomorphism class of $D_{0}$ without varying the curve $C_{0}$ as well, so that $\left(C_{0}, D_{0}\right)$ is an isolated point of the fiber $\varphi_{1}^{-1}\left(C_{0}\right)$ in $\Phi$. If in addition $D_{0} \in \Delta_{0}$ we will call $\left(C_{0}, D_{0}\right)$ a cusp preserving good degeneration of the family $X$; by what we have just said a family that admits a cusp preserving good degeneration will degenerate to $\Delta_{0}$ in codimension one in cusp preserving manner. Note that if $Y \subset X$ is a subfamily of curves, generically of the same genus $g$ and of having cusps at the same points where the general member of $X$ has them, and $Y$ admits a cusp preserving good degeneration, then $X$ does.

A basic observation is the following:

(4.3) Proposition. Let $W$ be a component of $V_{m, k}^{d, g}$. Then the following are equivalent:

(a) $W$ admits a cusp preserving good degeneration;

(b) $W$ degenerates to $\Delta_{0}$ in codimension one in cusp preserving manner, and

(c) $W$ contains a component of $U_{m, \kappa}^{d, g-1}$ of the type described in (4.1)(2), whose general member is irreducible.

Proof. Same as in $[\mathrm{H} 1,(4.1)]$.

Using this equivalence, we may deduce from $(4.1)$ as in $[\mathrm{H} 1,(4.2)]$ the following:

(4.4) Proposition. For $g \geq 1$, every component of $V_{m, k}^{d, g}$ admits a cusp preserving good degeneration.

Proof. Same as in $[\mathrm{H} 1,(4.2)]$ except that all curves (or some components of them) in $\mathbf{P}^{2}$ occurring in the proof have cusps at the fixed points $Q_{i}$ and their partial normalizations desingularize cusps. Q.E.D.

Now note that any general member of a component of $U_{1, \kappa}^{d . g-1}$ whose general member is irreducible is in fact a curve in a component $W^{\prime}$ of $V(d, \delta+1, \kappa)$. Since any component $W$ of $V(d, \delta, \kappa)$ contains in its closure a curve in $W^{\prime}$, we see that it contains in its closure $W^{\prime}$ from the fact that any component of $V(d, \delta+1, \kappa)$ is smooth and the local picture of $V(d, \delta, \kappa)$ at the point of $V(d, \delta+1, \kappa)$. By induction on genus any component $W$ of $V(d, \delta, \kappa)$ contains in its closure a component of $V\left(d, \frac{1}{2}(d-1)(d-2)-\kappa, \kappa\right)$.

\section{REFERENCES}

[ACGH] E. Arbarello, M. Cornalba, P. Griffiths and J. Harris, Geometry of curves, vol. 1, SpringerVerlag, New York, Heidelberg and Berlin, 1985.

[DH1] S. Diaz and J. Harris, Ideals associated to deformations of singular plane curves, Trans. Amer. Math. Soc. 309 (1988), 433-468.

[DH2] _ Geometry of the Severi variety, Trans. Amer. Math. Soc. 309 (1988), 1-34. 
[EH] D. Eisenbud and J. Harris, Divisors on general curves and cuspidal rational curves, Invent. Math. 74 (1983), 371-418.

[GH] P. Griffiths and J. Harris, Principles of algebraic geometry, Wiley-Interscience, New York, 1978.

[H1] J. Harris, On the Severi problem, Invent. Math. 84 (1986), 445-461.

[H2] _ Curves and their moduli, Notes of lectures given at the Amer. Math. Soc. Summer Institute, (1986), Preprint.

[H3] _ The genus of space curves, Math. Ann. 249 (1980), 191-204.

[H4] _ Families of smooth curves, Duke Math. J. 51 (1984), 409-419.

[Ha] R. Hartshorne, Algebraic geometry, Springer-Verlag, New York, Heidelberg and Berlin, 1977.

[Z1] O. Zariski, Algebraic surfaces, Springer-Verlag, Berlin, 1971.

[Z2] __, Algebraic system of plane curves, Amer. J. Math. 104 (1982), 209-226.

Department of Mathematics, University of Pennsylvania, Philadelphia, PennsylvaNIA 19104

Current address: Department of Mathematics, Pohang Institute of Science and Technology, Pohang, 790-330, Korea 